This is a post-print of the following article: Andric, Predrag; Curtin, W.A. Journal of the Mechanics and Physics of Solids 2018,, 144-161.. The formal publication is available at http://dx.doi.org/10.1016/j.jmps.2018.01.016 @ 2018. This manuscript version is made available under the CC-BY-NC-ND 4.0 license http://creativecommons.org/licenses/by-nc-nd/4.0/

\title{
New theory for crack-tip twinning in fcc metals
}

\author{
Predrag Andric, W.A. Curtin \\ Laboratory for Multiscale Mechanics Modeling \\ Ecole Polytechnique Federale de Lausanne \\ CH-1015 Lausanne, Switzerland
}

\begin{abstract}
Dislocation emission from a crack tip is a necessary mechanism for crack tip blunting and toughening. In fcc metals under Mode I loading, a first partial dislocation is emitted, followed either by a trailing partial dislocation ("ductile" behaviour) or a twinning partial dislocation ("quasibrittle"). The twinning tendency is usually estimated using the Tadmor and Hai extension of the Rice theory. Extensive molecular statics simulations reveal that the predictions of the critical stress intensity factor for crack tip twinning are always systematically lower (20-35\%) than observed. Analyses of the energy change during nucleation reveal that twin partial emission is not accompanied by creation of a surface step while emission of the trailing partial creates a step. The absence of the step during twinning motivates a modified model for twinning nucleation that accounts for the fact that nucleation does not occur directly at the crack tip. Predictions of the modified theory are in excellent agreement with all simulations that show twinning. Emission of the trailing partial dislocation, including the step creation, is predicted using a model recently introduced to accurately predict the first partial emission and shows why twinning is preferred. A second mode of twinning is found wherein the crack first advances by cleavage and then emits the twinning partial at the new crack tip; this mode dominates for emission beyond the first twinning partial. These new theories resolve all the discrepancies between the Tadmor twinning analysis and simulations, and have various implications for fracture behaviour and transitions.
\end{abstract}

Keywords: Cracks, Dislocations, Fracture, Molecular statics simulations 
This is a post-print of the following article: Andric, Predrag; Curtin, W.A. Journal of the Mechanics and Physics of Solids 2018,, 144-161.. The formal publication is available at http://dx.doi.org/10.1016/j.jmps.2018.01.016 @ 2018. This manuscript version is made available under the CC-BY-NC-ND 4.0 license http://creativecommons.org/licenses/by-nc-nd/4.0/

\section{Introduction}

Metals and alloys having an fcc structure (e.g. Al, $\mathrm{Cu}, \mathrm{Ni}, \mathrm{Ag}, \mathrm{Au}, \mathrm{Pd}, \mathrm{Pt}, \mathrm{Ir}$ ) are widely used structural materials in different industrial applications. For structural reliability, such materials must possess high fracture toughness. A minimum requirement for high fracture toughness is intrinsic ductility, achieved if an atomically sharp crack emits a dislocation prior to brittle cleavage [1]. When an fcc metal is intrinsically ductile, a first (leading) partial dislocation nucleates, moves to an equilibrium distance, and leaves a stacking fault extending back to the crack tip (See figure 1a). Ductile or quasi-brittle behaviour is then determined by the subsequent crack tip event. The three possible processes are:

(i) Emission of the trailing partial dislocation, nucleated on the same slip plane as the leading partial. The first and trailing partial dislocations combine to create a full dislocation having no trailing stacking fault, allowing the full dislocation to move far from the now-blunted crack tip (see Figure 1b); this behaviour is deemed "ductile".

(ii) Emission of the twinning partial dislocation having the same character as the first one but occurring on an immediately adjacent plane. This creates a two-layer twin embryo, with both partial dislocations remaining in the vicinity of the crack tip (see Figure 1c); this behaviour is deemed "quasi-brittle" since the process does not create far-field plasticity.

(iii) Emission of a partial dislocation having the same character as the first partial dislocation but occurring on a nonadjacent slip plane (see Figure 1d). Again, the emitted partial dislocation stays in vicinity of the crack tip, creating a region of two stable stacking faults separated by at least one plane of fcc atoms. This case is rare, and further increase of the applied load leads to another emission event that creates a three-layer twin embryo similar to what follows from process (ii); this behaviour is thus also deemed "quasi-brittle".

25 Understanding the fundamental competition between the processes of twinning and trailing partial emission thus provides insights into the controlling material parameters, which then contributes to ensuring ductile behaviour when designing new alloys [2].

Since a reliable model for predicting the intrinsic material fracture behaviour is a crucial step in achieving better performance, several theoretical models have been proposed for predicting the various critical stress intensity factors $K_{I e}$ for crack-tip dislocation emissions processes in metals at $\mathrm{T}=0 \mathrm{~K}$ [3-7]. The most widely used theoretical model for predicting the critical stress intensity factors $K_{I e}^{\text {first }}$ and $K_{I e}^{\text {trail }}$ for the emission of the first and trailing partial dislocations was proposed by Rice [8]. The solution for Mode II loading shows that the $K_{I I e}$ values depends on combinations of the unstable stacking fault energy $\gamma_{u s f}$, stable stacking fault energy $\gamma_{s s f}$, and material elastic properties. In Mode I, when the slip plane is inclined at an angle $\theta$, emission is 
This is a post-print of the following article: Andric, Predrag; Curtin, W.A. Journal of the Mechanics and Physics of Solids 2018,, 144-161.. The formal publication is available at http://dx.doi.org/10.1016/j.jmps.2018.01.016 () 2018. This manuscript version is made available under the CC-BY-NC-ND 4.0 license http://creativecommons.org/licenses/by-nc-nd/4.0/

assumed to occur by the same processes as in Mode II but correcting for the inclination of the slip plane. However, many molecular statics (MS) simulations revealed that fcc metals usually show crack-tip twinning at $\mathrm{T}=0 \mathrm{~K}$ [9]. This observation inspired Tadmor and Hai to propose an extension of the Rice theory for predicting the critical stress intensity factor for crack-tip twinning $K_{I e}^{\text {twin }}$ [10], showing that $K_{I e}^{\text {twin }}$ depends on the unstable twinning fault energy $\gamma_{u t f}$. By comparing the critical stress intensity factor for the emission of the trailing partial $K_{I e}^{\text {trail }}$ and the emission of the twinning partial dislocation $K_{I e}^{\text {twin }}$, twinning is preferred if $K_{I e}^{\text {twin }}<K_{I e}^{\text {trail }}$.

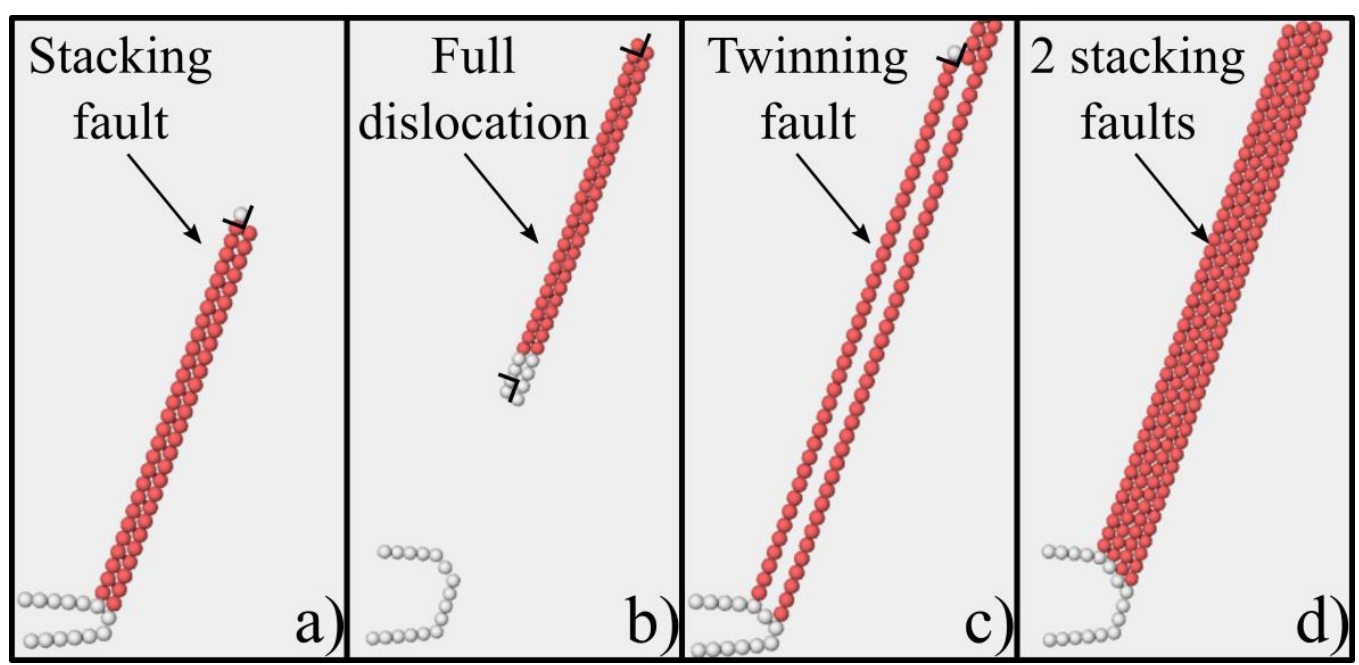

Figure 1: Partial dislocations emitted from a crack tip in a fcc metal: a) First partial dislocation at some equilibrium distance with the stacking fault behind it; b) First and trailing partial dislocation forming the full dislocation with the stacking fault between them; c) Twinning fault formed by the emission of the twinning partial dislocation; c) Region of two stacking faults formed by the second dislocation emission on the nonadjacent slip plane. Only non-fcc atoms are presented and coloured according to Common Neighbour Analysis [26]; red for hcp and white for other.

MD simulations have shown that these models are reasonable, but not highly accurate, for predicting the various $K_{I e}$. For the first partial dislocation emission, the creation of a surface step during emission was identified as a missing factor in the Rice theory. A number of authors incorporated a step energy into Rice-type theories but without obtaining significantly better predictions [11-14]. Recently, the present authors showed that the creation of the step fundamentally changes the emission process from being controlled by energy $\left(\gamma_{u s f}\right)$ to being controlled by a mechanical instability at the crack tip [15]. The new theory provides, qualitatively and quantitatively, reliable predictions for $K_{I e}^{\text {first }}$ and other details of the emission process not wellpredicted by the Rice theory.

The Tadmor-Hai model, based on the Rice approach, is quantitatively even less accurate for twinning tendencies. Also, the Tadmor twinning tendency predicts the emission of the trailing partial prior to twinning in some of materials while MS simulations always show twinning [16]. With the new insights into a completely different mechanism for the first emission process [15], the 
This is a post-print of the following article: Andric, Predrag; Curtin, W.A. Journal of the Mechanics and Physics of Solids 2018,, 144-161.. The formal publication is available at http://dx.doi.org/10.1016/j.jmps.2018.01.016 @ 2018. This manuscript version is made available under the CC-BY-NC-ND 4.0 license http://creativecommons.org/licenses/by-nc-nd/4.0/

processes of twinning and trailing emission of the second partial can now be revisited from a different perspective.

Here, we show that there is no creation of a surface step during the emission of the twinning partial. This suggests that the basic framework of Tadmor-Hai model for twinning is viable. We then show, however, that because the twinning slip plane does not intersect the crack front, the resolved shear stress along that plane at the location of the twin nucleation is lower than the shear stress along the trailing slip plane. Correcting for this difference by relating the far-field applied $K_{I}$ to the local shear displacement along the twinning plane leads to predictions in excellent agreement with the simulations, rectifying the previous deviations of 20-35\%. Using the same local analysis, we show that emission of the trailing partial dislocation is always accompanied by the creation of the surface step. Applying the new Andric-Curtin theory based on a crack tip instability due to the energy cost of forming the step to the case of trailing emission, we predict $K_{I e}^{\text {trail }}>K_{I e}^{\text {twin }}$ for all simulations, resolving the discrepancy between previous theory and simulation. Finally, we reveal an alternative mechanism of twinning wherein the crack first advances by cleavage and then emits the twinning partial, such that twinning is controlled by the Griffith cleavage fracture stress intensity $\mathrm{K}_{I c}$, where $K_{I e}^{\text {first }}<\mathrm{K}_{I c}<K_{I e}^{\text {twin }}$, in agreement with simulations where this process controls twinning. Furthermore, for subsequent emission of twinning partials after the first event, the twinning is often controlled by this alternative mechanism, thus connecting the process of extensive twinning directly to the process of cleavage fracture.

The reminder of this paper is organized as follows. In the first part of Section 2 we give a brief presentation of the Rice and Tadmor-Hai theories for predicting the critical stress intensity factor for various crack-tip dislocation emission processes. Then, in the remainder of Section 2, we evaluate the accuracy of both theories against atomistic simulations for 17 different fcc metals described with EAM potentials, and show the quantitative failures of the theories. To better understand the controlling crack-tip phenomena, in Section 3 we investigate the energy changes at the crack tip during the emission processes and show that twin partial emission is not accompanied by step creation while trailing partial emission does involve step creation. In the reminder of section 3, we introduce a modified Tadmor-Hai theory for predicting crack-tip twinning and show excellent agreement with the simulations. Then, we apply the Andric-Curtin theory to predict $K_{I e}^{\text {trail }}$ and resolve the discrepancy between the Tadmor-Hai trends and simulations. In Section 4 we introduce and analyse "forward" twinning as a new twinning mode. Finally, in Section 5, we discuss the implication of the new models developed here. 
This is a post-print of the following article: Andric, Predrag; Curtin, W.A. Journal of the Mechanics and Physics of Solids 2018,, 144-161.. The formal publication is available at http://dx.doi.org/10.1016/j.jmps.2018.01.016 @ 2018. This manuscript version is made available under the CC-BY-NC-ND 4.0 license http://creativecommons.org/licenses/by-nc-nd/4.0/

\section{Continuum theories of crack tip dislocation emission}

\subsection{Rice theory}

The Rice theory [8] is based on the Peierls concept [17] in which the periodic lattice shear resistance $\tau$ versus relative shear displacement $\Delta$ between two perfect crystalline blocks of material is $\tau_{g s f}=d \Psi_{g s f}^{\text {first }}(\Delta) / d \Delta$, where $\Psi_{g s f}^{\text {first }}(\Delta)$ is the so-called generalized stacking fault (GSF) energy. The GSF energy versus relative shear displacement $0 \leq \Delta \leq 2 b_{p}$, with $b_{p}$ being the partial Burgers vecor, is computed from atomistic simulations and is shown for fcc Ni in Figure 2 [18]. After emission of the first partial dislocation, there are two possible subsequent paths, twinning and trailing partial emission, that have different energies and different slip directions.

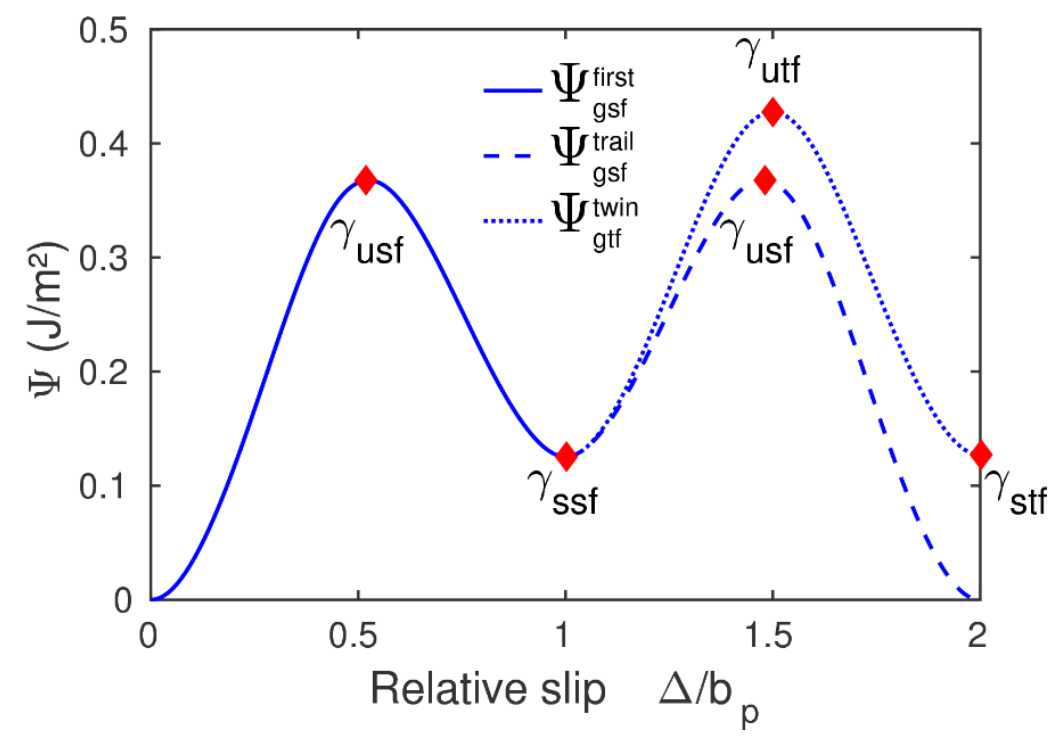

Figure 2: Generalized stacking fault (GSF) energy along the direction of the first partial dislocation (the solid line), GSF energy along the direction of the trailing partial dislocation (the dashed line), and generalized twinning fault (GTF) energy along the direction of the twinning partial dislocation (dotted line) computed in fcc $\mathrm{Ni}[18]$.

After the first emission, the first partial resides at some distance from the crack tip and imposes an additional shielding mixed-mode stress intensity acting at the crack tip. The crack tip responds to the total stress intensity at the crack tip, independent of how that stress intensity is established. Thus, the important physics of the problem remains at the crack tip, independent of the shielding due to the first partial. Therefore, in our restatements of the Rice and Hai-Tadmor theories here and in Sec. 2.2, we first neglect the effects of the first partial. Then in Sec. 2.3 we show that the shielding effect is the same for both processes and so does not influence which process is more favourable.

In Mode II loading, when the slip plane is coplanar with the crack, Rice introduced the displacement discontinuity $\delta=\Delta-h \tau_{g s f}(\Delta) / \mu$, where $h$ is the atomic inter-planar spacing and $\mu$ is the shear modulus for sliding along the slip plane, as the additional shear displacement beyond 
This is a post-print of the following article: Andric, Predrag; Curtin, W.A. Journal of the Mechanics and Physics of Solids 2018,, 144-161.. The formal publication is available at http://dx.doi.org/10.1016/j.jmps.2018.01.016 @ 2018. This manuscript version is made available under the CC-BY-NC-ND 4.0 license http://creativecommons.org/licenses/by-nc-nd/4.0/

the elastic response. An associated inelastic potential was then defined as $\Phi(\delta)=\Psi_{g s f}^{f i r s t}(\Delta)-$ $h \tau_{g s f}^{2}(\Delta) / 2 \mu$ which differs from $\Psi_{g s f}^{\text {first }}(\Delta)$ but has the same maximum value at the unstable stacking fault energy, $\max \left(\Psi_{g s f}^{\text {first }}(\Delta)\right)=\max (\Phi(\delta))=\gamma_{u s f}$ with $\delta=\Delta$. The path-independent

115 J-integral [19] then yields the macroscopic energy release rate $G$ for Mode II as $\Phi\left(\delta_{\text {tip }}\right)$,

$G=(1-v) K_{I I}^{2} / 2 \mu \equiv \Phi\left(\delta_{\text {tip }}\right)$.

Rice assumed that an incipient dislocation loses stability when the displacement discontinuity at the crack tip reaches the position of the unstable stacking fault energy $\delta_{c}^{t i p}=\Delta_{u s f}$. This point corresponds to the energy maximum $\gamma_{u s f}$ and, from Eq.1, yields the critical stress intensity factor for the emission in an isotropic elastic material as

$$
K_{I I e}=\sqrt{2 \mu \gamma_{u s f} /(1-v)} .
$$

For dislocation emission in Mode I, where the slip plane is inclined at an angle $\theta$ with respect to the crack plane, the J-integral cannot be applied. Rice thus assumed that the slip profile along the sliding plane in Mode I is the same as that in Mode II. This enables application of the result for Mode II to Mode I simply by using the correct resolved shear stress along the slip plane, leading to

$$
K_{I e}=\sqrt{2 \mu \gamma_{u s f} /(1-v)} / \cos ^{2}(\theta / 2) \sin (\theta / 2)
$$

125 with emission again occurring at $\delta_{c}^{t i p}=\Delta_{u s f}$. The results of Eq. 2 and 3 can be generalized to full anisotropic elasticity [20] as

$$
\begin{aligned}
& K_{I I e}=\sqrt{\gamma_{u s f} o\left(\theta, \varphi_{\text {first }}\right)} \\
& K_{I e}=\sqrt{\gamma_{u s f} o\left(\theta, \varphi_{\text {first }}\right)} / F_{12}(\theta),
\end{aligned}
$$

where $o\left(\theta, \varphi_{\text {first }}\right)$ is a material elastic property computed from Stroh tensor [21] as a function of the slip plane inclination angle $\theta$, and the angle $\varphi_{\text {first }}$ between the dislocation Burgers vector and the crack front direction, and $F_{12}(\theta)$ corrects for the resolved shear stress along the slip plane. For more details see Appendix A.

With increasing far-field loading, the first partial dislocation can be followed either by (i) the trailing partial dislocation emission or (ii) the twinning partial dislocation emission. A model for the critical stress intensity factor for trailing partial emission $K_{I e}^{\text {trail }}$ was introduced by Rice [8]. While similar to the analysis for the leading partial, additional important aspects enter. As the first partial dislocation moves away from the crack tip, it leaves behind a stacking fault, as shown in 
This is a post-print of the following article: Andric, Predrag; Curtin, W.A. Journal of the Mechanics and Physics of Solids 2018,, 144-161.. The formal publication is available at http://dx.doi.org/10.1016/j.jmps.2018.01.016 @ 2018. This manuscript version is made available under the CC-BY-NC-ND 4.0 license http://creativecommons.org/licenses/by-nc-nd/4.0/

Figure 1a. This causes the energy at the crack tip to be shifted to the intrinsic stacking fault $\gamma_{s s f}$ (see Figure 2). The trailing partial nucleates on the same slip plane as the leading partial, and so the energy barrier for its emission is $\left(\gamma_{u s f}-\gamma_{s s f}\right)$ (dashed line in Figure 2a). The emission of the trailing partial dislocation is also associated with a change in the angle between the partial dislocation Burgers vector and the crack front direction, $\varphi_{\text {first }} \rightarrow \varphi_{\text {trail }}$. In fcc metals, the difference is always $\left|\varphi_{\text {trail }}-\varphi_{\text {first }}\right|=60^{\circ}$. By using the constrained path approximation and the same assumption regarding slip profiles in Mode II and Mode I, Rice derived the critical stress intensity factor at the tip for the emission of the trailing partial dislocation in Mode I as

$$
K_{\text {Ie,tip }}^{\text {trail }}=\sqrt{\left(\gamma_{u s f}-\gamma_{s s f}\right) o\left(\theta, \varphi_{\text {trail }}\right)} / F_{12}(\theta) \cos \left(\varphi_{\text {trail }}\right)
$$

\subsection{Tadmor-Hai theory for crack-tip twinning}

The first partial dislocation can also be followed by the emission of the twinning partial dislocation. An analytical model for predicting $K_{I e}^{t w i n}$ was proposed by Tadmor and Hai [10] as an extension of the Rice theory with two main differences: (i) the angle between the twinning partial Burgers vector and the crack front direction is same as for the first partial dislocation $\varphi_{\text {twin }}=$ $\varphi_{\text {first }}$, and (ii) the twinning partial forms the micro-twin boundary by nucleation on the adjacent slip plane and, therefore, the energy functional describing the shear displacement along the twinning plane is the generalized twinning fault (GTF) energy (the dotted line in Figure 2). The energy barrier for the twinning partial emission is then $\left(\gamma_{u t f}-\gamma_{s s f}\right)$. Following Rice, Tadmor and

155 Hai derived the critical stress intensity factor at the tip for the emission of the twinning partial dislocation as

$$
K_{\text {Ie,tip }}^{\text {twin }}=\sqrt{\left(\gamma_{u t f}-\gamma_{s s f}\right) o\left(\theta, \varphi_{\text {first }}\right)} / F_{12}(\theta) .
$$

At temperature of $T=0 \mathrm{~K}$ a material is predicted to emit the twinning partial dislocation when $K_{I e, t i p}^{\text {twin }}<K_{\text {Ie,tip }}^{\text {trail }}$

\subsection{Shielding effect due to the first partial dislocation}

After nucleation, the first partial dislocation moves to an equilibrium distance $r$ along the slip plane ahead of the crack, at which point the total Peach-Koehler force is zero [8]. The PeachKoehler force has three main contributions: (i) the force due to the applied $K_{I}$, resolved for the particular slip plane inclination angle, which drives the dislocation away from the crack tip, (ii) the stacking fault which attracts the dislocation back towards the crack tip, and (iii) the image force, 
This is a post-print of the following article: Andric, Predrag; Curtin, W.A. Journal of the Mechanics and Physics of Solids 2018,, 144-161.. The formal publication is available at http://dx.doi.org/10.1016/j.jmps.2018.01.016 @ 2018. This manuscript version is made available under the CC-BY-NC-ND 4.0 license http://creativecommons.org/licenses/by-nc-nd/4.0/

due to crack free surface, which also draws the dislocation back toward the crack tip. Each term can be evaluated precisely within linear elasticity, leading to the equilibrium condition

$$
\frac{K_{I} F_{12}(\theta) b_{p}}{\sqrt{2 \pi r}}-\gamma_{s s f}-\frac{b_{p}^{2} o\left(\theta, \varphi_{\text {first }}\right)}{8 \pi r}=0 .
$$

which yields the equilibrium distance $r$ versus applied $K_{I}$ as

$$
r=\frac{\left(K_{I} F_{12}(\theta)\right)^{2} b_{p}^{2}}{8 \pi \gamma_{s s f}^{2}}\left(1+\sqrt{1-\frac{\gamma_{s s f} o\left(\theta, \varphi_{\text {first }}\right)}{\left(K_{I} F_{12}(\theta)\right)^{2}}}\right)^{2}
$$

where the exact solution is the largest root.

The first partial dislocation then shields the crack tip, reducing the crack tip stress intensity factor $[6,23]$. Specifically, a dislocation at distance $r$ along a slip plane inclined at angle $\theta$ in an anisotropic elastic material generates additional stress intensity factors $k_{I}^{d}$ and $k_{I I}^{d}$, given by [24]

$$
\begin{aligned}
& k_{I}^{d}=-\frac{1}{2 \sqrt{2 \pi r}} \sum_{j=1}^{3} \Lambda_{2 j}^{-1} D_{2 k}^{\mathrm{j}}(\theta) b_{k} \\
& k_{I I}^{d}=-\frac{1}{2 \sqrt{2 \pi r}} \sum_{j=1}^{3} \Lambda_{1 j}^{-1} D_{2 k}^{\mathrm{j}}(\theta) b_{k}
\end{aligned}
$$

with $\boldsymbol{\Lambda}$ being the Stroh energy tensor, $b_{k}$ the Burgers vector, and $\boldsymbol{D}^{j}(\theta)$ related to the angular distribution of the stresses near the crack tip for the particular $j$ mode of loading given by

$$
\sigma_{k l}(r, \theta)=\sum_{j=1}^{3} \frac{1}{\sqrt{2 \pi r}} K_{j} D_{k l}^{j}(\theta) .
$$

175 These additional stress intensity factors then shift the far-field stress intensity factor $K_{I}$ for the emission of the trailing or twinning partial to

$$
\begin{aligned}
& K_{I e}^{\text {trail }}=\sqrt{\left(\gamma_{u s f}-\gamma_{s s f}\right) o\left(\theta, \varphi_{\text {trail }}\right)} / F_{12}(\theta) \cos \left(\varphi_{\text {trail }}\right)+k_{I}^{d}+\frac{F_{22}(\theta)}{F_{12}(\theta)} k_{I I}^{d} \\
& K_{I e}^{\text {twin }}=\sqrt{\left(\gamma_{u t f}-\gamma_{s s f}\right) o\left(\theta, \varphi_{\text {first }}\right)} / F_{12}(\theta)+k_{I}^{d}+\frac{F_{22}(\theta)}{F_{12}(\theta)} k_{I I}^{d}
\end{aligned}
$$

with $F_{22}(\theta)$ correcting for the resolved shear stress along the slip plane due to $k_{I I}^{d}$. We note that the emission of the second partial dislocation is only controlled by the processes in the vicinity of the crack-tip. As noted above, the shielding affects both processes equally through a net stress intensity acting at the crack tip. Shielding is thus not intrinsic to either of the two possible crack-tip processes, and does not affect which process is more favourable. 
This is a post-print of the following article: Andric, Predrag; Curtin, W.A. Journal of the Mechanics and Physics of Solids 2018,, 144-161.. The formal publication is available at http://dx.doi.org/10.1016/j.jmps.2018.01.016 @ 2018. This manuscript version is made available under the CC-BY-NC-ND 4.0 license http://creativecommons.org/licenses/by-nc-nd/4.0/

\subsection{Atomistic assessment of the continuum theories}

To evaluate the above theories for the various critical stress intensity factors $K_{I e}$ for cracktip dislocation emission, we execute molecular statics simulations using the Large-scale Atomic/Molecular Massively Parallel Simulator (LAMMPS) [25]. For all cases, we model a semiinfinite crack under plane strain loading conditions with periodic boundary conditions along the crack front direction ( $\mathrm{z}$ ) with the crack lying in $\mathrm{x}-\mathrm{z}$ plane and crack tip at $\mathrm{y}=0$. The simulation cell has dimensions of approximately $150 \times 150 \times 1 \mathrm{~nm}$. In homogeneous materials at $\mathrm{T}=0 \mathrm{~K}$, as long as there is an integer number of the crystal lattice periods along the $\mathrm{z}$ direction, the dislocation emission is independent of the cell thickness. An fcc crystal is oriented with $X=[\overline{1} \overline{1} 2], Y=[111]$ and $Z=[\overline{1} 10]$, forming a crack with a single slip plane inclined at the angle $\theta=70.53^{\circ}$ to the crack and with the Burgers vector angle $\varphi_{\text {first }}=0^{0}$; this is usually the orientation for easiest dislocation emission. The crack is loaded by incrementing the atomic displacements according to the anisotropic displacement field for linear elastic fracture mechanics (LEFM), corresponding to the increments of the applied stress intensity $K_{I}$ (see Appendix A). After each loading increment of $\Delta K_{I}=0.001 \mathrm{MPa} \sqrt{\mathrm{m}}$, the boundary atoms within $2 r_{c}$ ( $r_{c}=$ cut-off distance of the inter-atomic potential) of the simulation cell outer boundary are held fixed while the all other atoms are relaxed to minimize the system energy using the conjugate gradient method. We terminate the simulation after dislocation emission occurs.

At loads below the Griffith value $K_{I c}$, an atomically sharp crack is unstable to closure - the traction free crack surface (the continuum representation of the crack) cannot be simply imposed on atoms. There are several approximate approaches to address this issue. Here, we artificially delete the inter-atomic interactions between the crack surfaces ("screening"), which we have previously found is the best representation of a sharp crack. We analyse 17 different fcc systems described by EAM interatomic interactions (Aluminum [18] [27]; Copper [28]; Nickel [18]; Au, Ag, Pd [29], and a set homogenized $\mathrm{Fe}_{(1-\mathrm{x}) / 2} \mathrm{Ni}_{(1-\mathrm{x}) / 2} \mathrm{Cr}_{\mathrm{x}}$ labelled by $\mathrm{Cr}$ content as $\mathrm{Cr} 10-\mathrm{Cr} 100$ [30] [31]).

Figure $3 \mathrm{a}$ shows the critical stress intensity factor for the emission of the first partial

210 dislocation $K_{I e}^{\text {first }}$ as predicted by the Rice theory and as measured in the simulations. The Rice theory is systematically lower than simulation results and do not follow the detailed trends. For some materials $(\mathrm{Ni}, \mathrm{Cr} 10)$, the Rice prediction is accurate while for other materials ( $\mathrm{Au}, \mathrm{Pd}$ and $\mathrm{Cu}$ ) the differences are up to 50\%. In addition, as presented previously and not shown here, the critical shear displacement $\Delta_{1}^{c}$ at the crack tip just before emission is generally much smaller than the Rice 215 prediction. These inconsistencies exist because the Rice theory does not account for the creation of a surface step during the dislocation emission. We recently proposed a new theory, summarized 
This is a post-print of the following article: Andric, Predrag; Curtin, W.A. Journal of the Mechanics and Physics of Solids 2018,, 144-161.. The formal publication is available at http://dx.doi.org/10.1016/j.jmps.2018.01.016 @ 2018. This manuscript version is made available under the CC-BY-NC-ND 4.0 license http://creativecommons.org/licenses/by-nc-nd/4.0/

below, to account for the surface step, leading to good agreement with nearly all simulations (see Figure 3a).
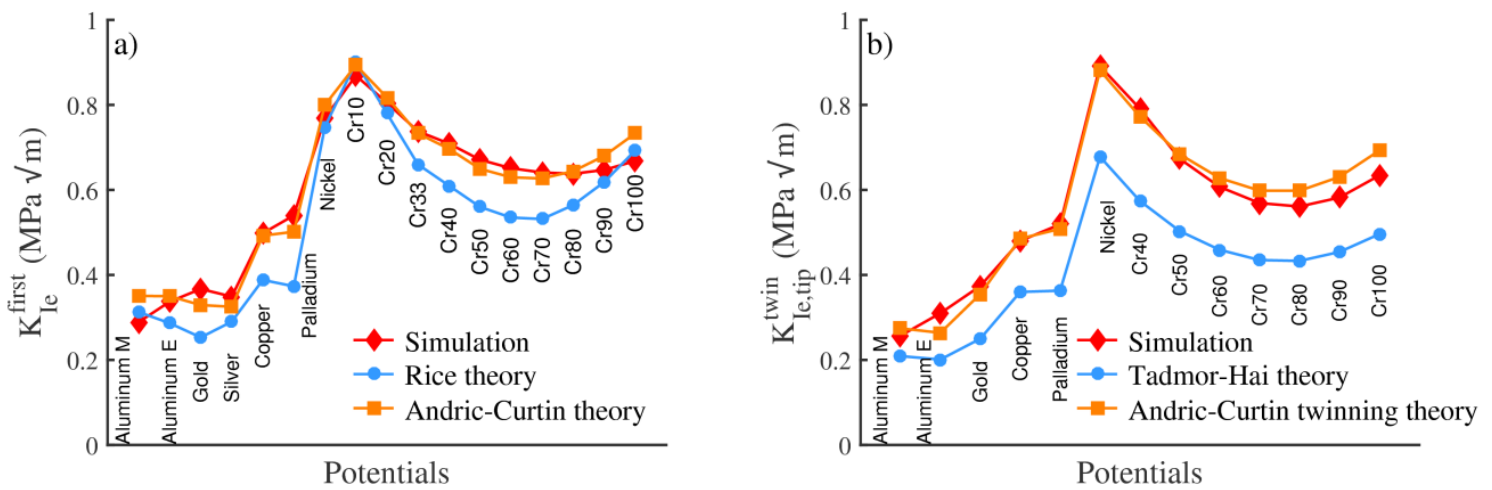

Figure 3: Critical stress intensity factor for emission as observed in the simulations (red diamonds), along with the predictions of continuum theories based on the Peierls model (blue circles), and the Andric-Curtin theory (orange squares) for a) the first partial dislocation $K_{I e}^{\text {first }}$ and b) the "back" twinning partial dislocation $K_{\text {Ie,tip }}^{\text {twin }}$.

We now examine the second partial dislocation emission by performing an additional set of simulations, as follows. To capture only the crack tip effects without the shielding due to the first partial dislocation we first displace the part of the non-loaded perfect crystal, marked with the red line (see Figure 4), along the leading partial slip direction by the partial Burgers vector $b_{p}=$ $a_{0} / \sqrt{6}$, where $a_{0}$ is a lattice parameter. This method is equivalent to actually nucleating the partial, moving it to infinity by adding a dipole of infinite spacing, and then unloading the crystal to zero load. Since the crack surfaces are already formed by screening of interactions, the crystal is stable in this new initial configuration. We then perform the standard K-test as described above. We will consider results including the shielding effects later. In every materials studied, we observe twinning. In contradiction with the simulations, the Tadmor-Hai theory predicts emission of the trailing partial prior to emission of the twinning partial for $\mathrm{Al} \mathrm{M}, \mathrm{Cr} 100$ and $\mathrm{Cr} 90$, both with and without the shielding effect. Since the shielding effect only changes the stress intensity acting on the crack tip, and not the critical values for the two processes, this is not a surprising result. The discrepancy for Al M was noted by Yamakov et al. [16]. Furthermore, the simulations show two different twinning mechanisms. As shown in Figure 5b, one mechanism is twinning partial emission by sliding along the adjacent slip plane behind the leading partial slip plane (denoted "back" twinning). As shown in Figure 5c, a second mechanism forms the micro-twin by crack advancement of one lattice spacing in [ $\overline{1} \overline{1} 2]$ direction followed by sliding along the adjacent plane in front of the leading partial (denoted "forward" twinning). The "forward" twinning occurs in Ag, and Cr10Cr33, while all other materials tested show "back" twinning. These two different twinning modes were first observed in atomistic simulations of Hai and Tadmor [9]. However, the later Tadmor-Hai theory [10] does not distinguish between these two physically different events. Because "forward" 
This is a post-print of the following article: Andric, Predrag; Curtin, W.A. Journal of the Mechanics and Physics of Solids 2018,, 144-161.. The formal publication is available at http://dx.doi.org/10.1016/j.jmps.2018.01.016 @ 2018. This manuscript version is made available under the CC-BY-NC-ND 4.0 license http://creativecommons.org/licenses/by-nc-nd/4.0/

twinning resemble Griffith cleavage followed by dislocation emission, the Tadmor-Hai model is essentially a model for "back" twinning.

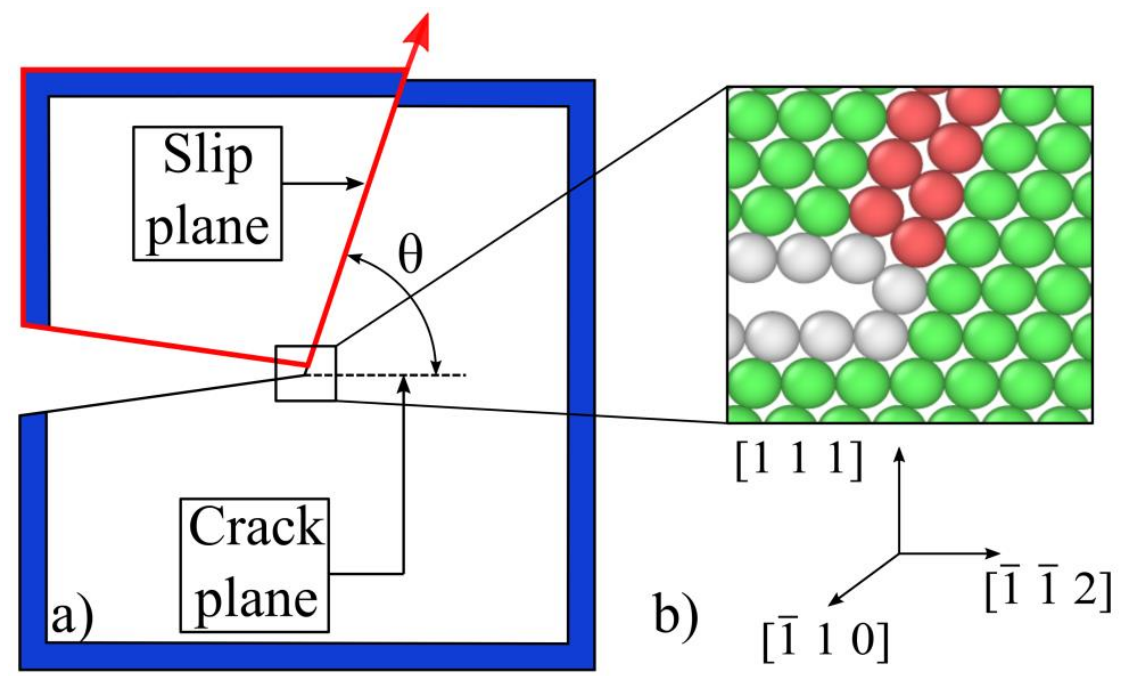

Figure 4: a) Geometry used to simulate second partial dislocation emission using a semi-infinite crack with the first partial dislocation removed to infinity, achieved by displacing the region marked by the red line by the first partial Burgers vector. Blue color indicates boundary atoms. b) Atomic scale crack-tip geometry at zero loading. Atoms are colored based on Common Neighbour Analysis [26]; green for fcc, red for hcp and white for surface.

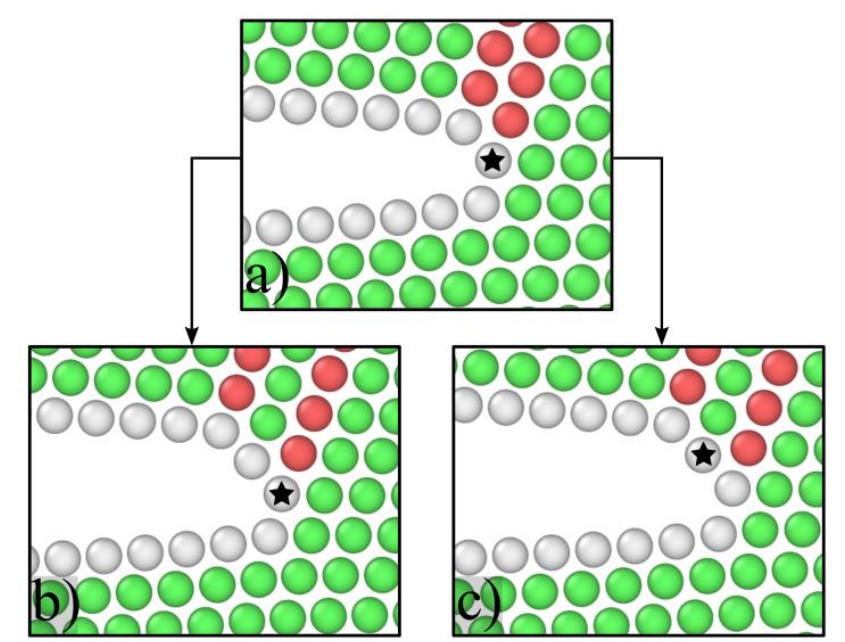

Figure 5: a) Crack geometry in Mode I at the critical load for the emission of the twinning partial dislocation. b) Crack geometry after "back" twinning emission. c) Crack geometry after "forward" twinning emission. The same atom at the original crack tip is indicated in all figures to show the two different mechanisms.

Figure $3 \mathrm{~b}$ shows the simulated critical stress intensity factor $K_{I e, t i p}^{t w i n}$ along with the TadmorHai predictions (Eq.6) for all "back" twinning cases. Figure $3 b$ shows that the predictions are systematically lower than the simulations by 20-35\%. However, the theoretical predictions do follow the trend observed in the simulations, unlike the situation for the first partial dislocation emission (Figure 3a). The systematic and significant deviation of the Tadmor-Hai theory requires the development of a new theory. Following our recent discrete analysis of the first partial emission, below we examine the detailed local processes and location of the actual nucleation event. The 
This is a post-print of the following article: Andric, Predrag; Curtin, W.A. Journal of the Mechanics and Physics of Solids 2018,, 144-161.. The formal publication is available at http://dx.doi.org/10.1016/j.jmps.2018.01.016 @ 2018. This manuscript version is made available under the CC-BY-NC-ND 4.0 license http://creativecommons.org/licenses/by-nc-nd/4.0/

analysis reveals both the source of the discrepancy with previous models and motivates a new model that quantitatively captures the observed behaviour (see Figure 3b).

\section{Discrete analysis of crack tip dislocation emission}

\subsection{Andric-Curtin theory for the first partial emission}

The inconsistencies and inaccuracy in the Rice theory arise because that theory does not account for the creation of the surface step at the crack tip upon emission in Mode I at angle $\theta$. The step energy is revealed by examining the energy changes in each discrete structural unit along the slip plane during slip (see Figure 6 for Ni). The energy change of the first unit at the crack tip (called the nucleation energy $\Psi_{\text {nuc }}^{\text {first }}(\Delta)$ ) differs significantly from the GSF energy due to the creation of a surface step. $\Psi_{\text {nuc }}^{\text {first }}(\Delta)$ also does not have a maximum, which precludes the application of the Rice theory.

We summarize here our recent theory for $K_{I e}^{\text {first }}$ that accounts for the creation of the surface step [15]. Because shearing of the crack tip unit requires high energy, the shear resistance $\tau_{\text {res }}=d \Psi_{\text {nuc }}^{\text {first }}(\Delta) / d \Delta$ is also very high (see Figure 6 for Ni). The nascent dislocation is "trapped" at the first crack tip and can only be emitted once the crack tip unit reaches a point of mechanical instability. As shown in Figure 7a for a sinusoidal Peierls-type model for $270 d \Psi_{\text {nuc }}^{\text {first }}(\Delta) / d \Delta$, the point of mechanical instability is reached at the critical displacement $\Delta_{1}^{c}$ where the shear resistance $\tau_{\text {res }}$ of the crack tip unit cannot balance the local driving shear stress $\tau_{a p p}=\tau_{a p p, 0}\left(K_{I}\right)-\mu \Delta / h(\beta-1)$, which is a linear function of the crack tip displacement and with $\tau_{a p p, 0}\left(K_{I}\right)$ lattice. In contrast (Figure $7 \mathrm{a}$ ), when the crack tip resistance is provided only by the GSF energy, a mechanical instability does not occur until the critical displacement reaches the much larger value of $\Delta_{1}^{c}=b_{p} / 2$, and this is achieved at much lower applied $K_{I}$ and much lower $\tau_{a p p, 0}\left(K_{I}\right)$. In the presence of the extra step energy, the critical stress energy for emission can be expressed relative to the Rice value as

$$
K_{\text {Ie,step }}^{\text {first }} / K_{\text {Ie,Rice }}^{\text {first }}=\tau_{a p p, 0}^{c, \text { step }} / \tau_{a p p, 0}^{c, \text { Rice }}
$$

where the critical stresses $\tau_{a p p, 0}^{c, s t e p}$ and $\tau_{a p p, 0}^{c, \text { Rice }}$ are shown in Figure 7a. When the step energy is sufficiently high $\left(\gamma_{s t e p} \gg \gamma_{u s f}\right)$, the new theory predicts that $K_{I e}^{\text {first }}$ is larger than the Rice value 280 but at a smaller critical shear displacement $\Delta_{1}^{c}<b_{p} / 2$. The new analysis conceptually resolves the discrepancy between the Rice theory and simulations. 
This is a post-print of the following article: Andric, Predrag; Curtin, W.A. Journal of the Mechanics and Physics of Solids 2018,, 144-161.. The formal publication is available at http://dx.doi.org/10.1016/j.jmps.2018.01.016 @ 2018. This manuscript version is made available under the CC-BY-NC-ND 4.0 license http://creativecommons.org/licenses/by-nc-nd/4.0/

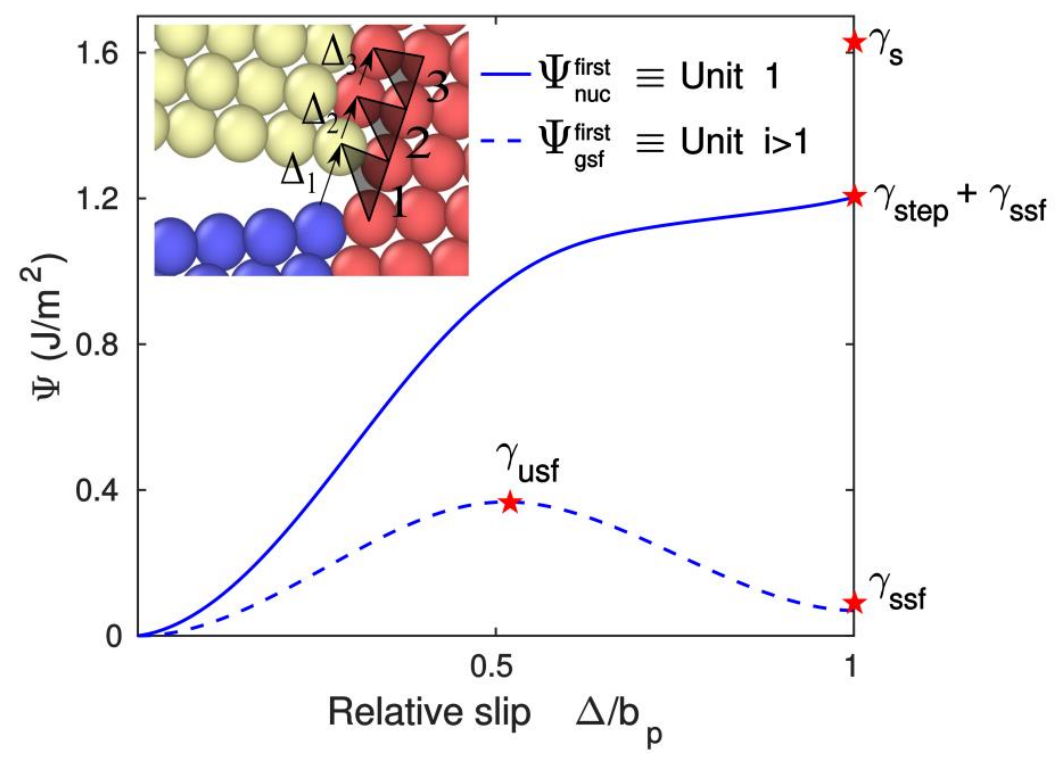

Figure 6: Slip energy of structural units (see inset) along the first partial slip plane. Energy of the crack-tip unit (solid line) is much higher than all other units (dashed line), which follow the bulk GSF curve. Results here are for fcc Ni [18].
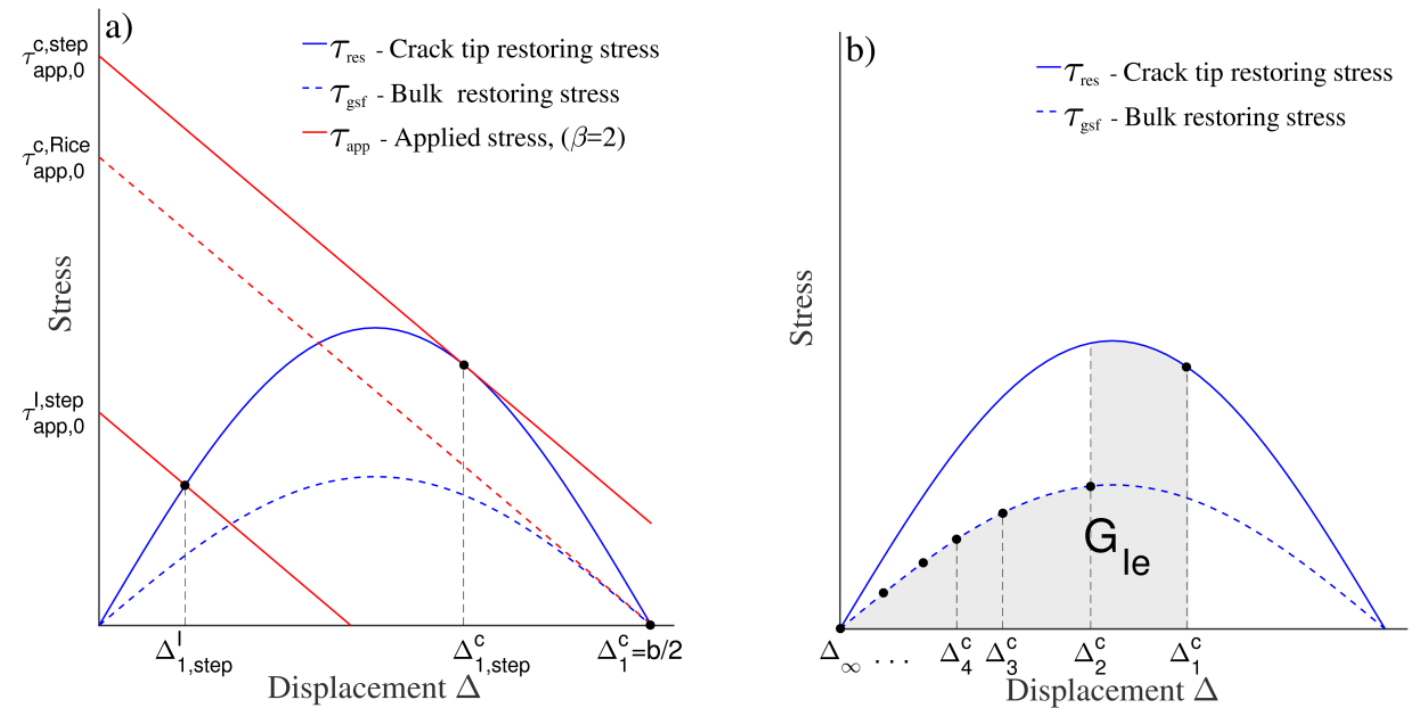

Figure 7: a) Graphical construction for crack-tip unit equilibrium displacement $\Delta_{1}$ prior to the emission of the first partial dislocaiton in Mode I loading when a step is created. All other shear displacements along the sliding plane are assumed in the linear regime. Solid blue line: crack-tip shear resistance, including the step energy; red lines: applied stress $\tau_{a p p}$ generated by the surrounding elastic material as a function of the shear displacement $\Delta$ for several applied stress intensity values; dashed blue line: lattice restoring shear stress in the bulk material, for reference. b) Shear displacements of structural units along the slip plane at the point of first partial dislocation nucleation when non-linear behavior is present further along the sliding plane. The shaded area corresponds to the critical energy release rate for dislocation emission.

The new theory, in the simple form above, assumes that all non-linear behaviour is confined 285 only to the crack tip unit, with all other units along the slip plane in the elastic regime. In general, there exists some non-linear behavior further along the slip plane, where the crack units follow the usual GSF energy. The energy required to shear the crack tip unit up to the critical displacement $\Delta_{1}^{c}$ can then be calculated from $\Psi_{\text {nuc }}^{\text {first }}(\Delta)$ and $\Psi_{g s f}^{\text {first }}(\Delta)$ as the shaded area shown in Figure $7 \mathrm{~b}$. In 
This is a post-print of the following article: Andric, Predrag; Curtin, W.A. Journal of the Mechanics and Physics of Solids 2018,, 144-161.. The formal publication is available at http://dx.doi.org/10.1016/j.jmps.2018.01.016 @ 2018. This manuscript version is made available under the CC-BY-NC-ND 4.0 license http://creativecommons.org/licenses/by-nc-nd/4.0/

terms of the two critical displacements $\Delta_{1}^{c}$ and $\Delta_{2}^{c}$ of the first and second crack-tip units, the critical energy release rate at emission $J=G_{I e}$ is

$J=\Psi_{\text {nuc }}^{\text {first }}\left(\Delta_{1}^{c}\right)-\Psi_{\text {nuc }}^{\text {first }}\left(\Delta_{2}^{c}\right)+\Psi_{g s f}^{\text {first }}\left(\Delta_{2}^{c}\right) \equiv G_{I e}^{\text {first }}$

and $K_{I e}^{\text {first }}$ follows as

$$
K_{I e}^{\text {first }}=\sqrt{G_{\text {Ie }}^{\text {first }}\left(\theta, \varphi_{\text {first }}\right)} / F_{12}(\theta) .
$$

Identifying the critical shear displacements $\Delta_{1}^{c}, \Delta_{2}^{c}$ in the non-linear case is complicated, but extensive simulations reveal typical values across a wide range of fcc systems [15]. Use of the typical values enabled us to development an excellent approximate analytic formula for $G_{I e}^{\text {first }}$ only in terms of the slip plane surface energy $\gamma_{s}$ and the unstable stacking fault energy $\gamma_{u s f}$ as

$$
G_{\text {Ie }}^{\text {first }}=\left\{\begin{array}{c}
0.145 \gamma_{s}+0.5 \gamma_{u s f}, \gamma_{s}>3.45 \gamma_{u s f} \\
\gamma_{u s f}, \quad \gamma_{s}<3.45 \gamma_{u s f}
\end{array}\right.
$$

Figure 3a shows the critical stress intensity factor for the emission of the first partial dislocation computed according to Eqs.14-15; excellent agreement with the simulation results is found in all cases.

The above results show that a very detailed discrete analysis of the crack tip deformation processes enables for the identification of the main physical aspects that control the first dislocation emission. Below, we use the same approach to understand (i) the simulated critical stress intensity factor for emission of the second partial dislocation, (ii) why the second partial is always the twinning partial, and (iii) when "back" twinning and "forward" twinning occur. We will thereby rectify the discrepancies between simulations and previous theories.

\subsection{Energy changes near the crack-tip during second partial dislocation emission}

We start by investigating the energy changes during slip in the structural units near the crack tip that are relevant for the emission of twinning and trailing partial dislocation. A schematic of the discrete analysis is shown in Figure 8a, showing two adjacent discrete slip planes surrounded by an elastic material containing a crack. The two slip planes are further envisioned to be composed of individual structural units defined by specific triads of atoms, as indicated. Figure $8 \mathrm{~b}$ shows a closeup atomistic view of the crack tip region, and specifically identifies the three structural units that are crucial to our subsequent analysis: one unit directly at the crack tip relevant for trailing partial emission and two units along the nascent twinning plane.

Atom-by-atom energy changes are measured along the two possible slip planes in a manner similar to that proposed by Zamora et al. [32]. Identifying such atomic energies are only possible 
This is a post-print of the following article: Andric, Predrag; Curtin, W.A. Journal of the Mechanics and Physics of Solids 2018,, 144-161.. The formal publication is available at http://dx.doi.org/10.1016/j.jmps.2018.01.016 @ 2018. This manuscript version is made available under the CC-BY-NC-ND 4.0 license http://creativecommons.org/licenses/by-nc-nd/4.0/

when using interatomic potentials where the total energy is represented as a sum of atomic energies. More complex approaches would be needed to localize the measured total energy changes when computed using quantum mechanical methods; Zamora et al. have headed in such a direction for the specific problem of step energies [32]. First, we define the non-orthogonal computational cell oriented with $X=[\overline{1} \overline{1} 2], \quad Y=[111]$ and $Z=[\overline{1} 10]$, and with dimensions of $10 \sqrt{6} a_{0} \times 12 \sqrt{3} a_{0} \times 2 \sqrt{2} a_{0} A$. We set periodic boundary conditions in the [112] and [110] directions with a free surface in the [11i ] direction. We delete a group of atoms to simulate the free surface close to the crack tip and then define the domain of analysis as shown in Figure 9a. The domain is sufficiently long in the [112] direction that atoms far from the crack tip and/or free surface behave like bulk fcc atoms. The initial configuration for computation of the atomic energy change is the completely relaxed crystal containing a stacking fault (see Figure 9a). We then rigidly slide a portion of the left part of the crystal in the desired slip direction. When the sliding plane is the twin plane, sliding is continued until the full two-layer twinning fault is formed. When the sliding plane is the original stacking fault plane, sliding is continued until the stacking fault is eliminated, which represents the emission of the trailing partial dislocation. During sliding, we compute the energy change of each individual atom in the domain as $\delta E^{\text {atom }}(\Delta)=\mathrm{E}^{\text {atom }}(\Delta)-$ $\mathrm{E}^{\text {atom }}(0)$, where $E^{\text {atom }}(\Delta)$ is the atomic energy at slip displacement magnitude $\Delta$ in the slip direction. During sliding, atomic relaxations are only allowed in the direction normal to the slip plane, as in standard GSF/GTF computations.

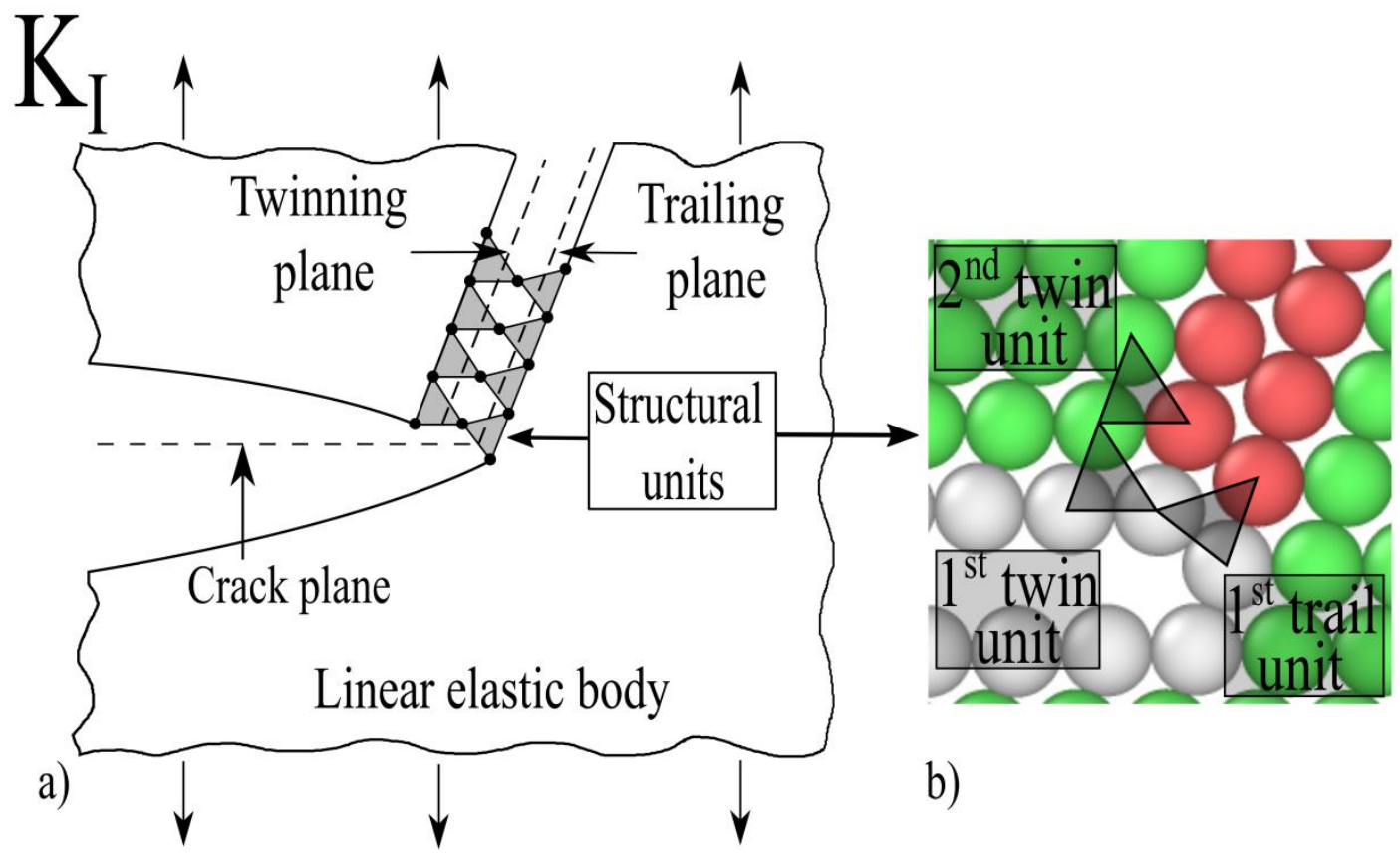

Figure 8: a) Linear elastic body with semi-infinite crack under Mode I loading with embedded slip planes for analysis of the emission of the trailing and twinning partial dislocations. The slip planes are characterized by atomic structural units. b) The three structural units that control emission of the second partial dislocation. 


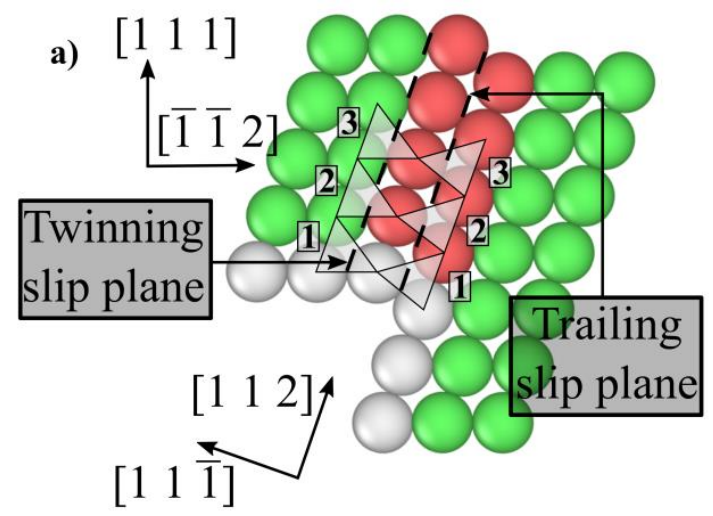

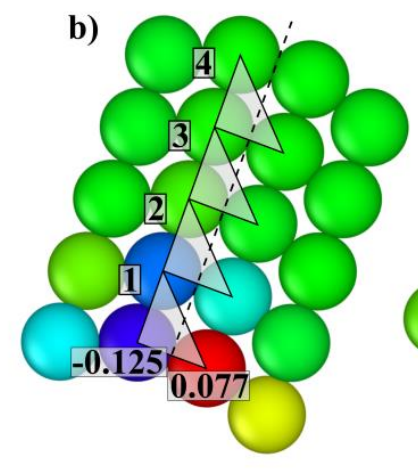

Twinning units

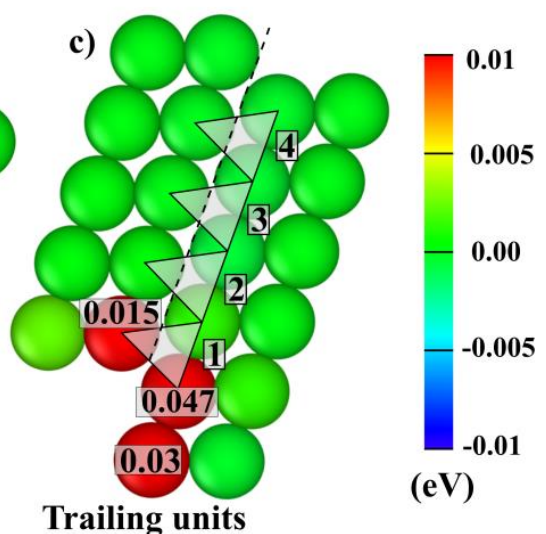

Trailing units

Figure 9: a) Initial configuration of the computational domain used to compute atom-by-atom energy changes due to relative shear displacement $\Delta$ along the trailing or twinning slip plane. Energy change per atom after subtraction of the energy changes per atom corresponding to the bulk GSF and GTF energies per atom, showing the energy changes due to surface effects near the crack tip for b) the twinning partial and c) the trailing partial, at relative slip $\Delta=b_{p} / 2$. Atoms in (a) are colored by Common Neighbour Analysis [26] (green for fcc, red for hcp and white for surface); Atoms in (b) and (c) are colored according to energy change shown by the scale. Energies of atoms near the crack tip are off scale, and indicated in each figure.

The crack-tip dislocation emission influences the energies only near the crack tip, for both twinning and trailing partial emission. This is demonstrated by subtracting the atom-by-atom energy changes for the GSF and GTF sliding from the measured $E^{\text {atom }}(\Delta)$, as shown in Figure $9 \mathrm{~b}$ and c, at shear displacement $\Delta=b_{p} / 2$. For both twinning partial and the trailing partial emission, (i) the energy change of the crack-tip/surface atoms differs significantly from the response away from the crack-tip/surface and (ii) the energy changes relative to bulk response are almost negligible immediately away from the crack-tip/ surface. The per-atom energy change is crucial, but not sufficient to provide insights into the emission process. Therefore, we examine the energy change of the basic structural units along the slip plane as indicated in Figure 9. We compute the energy change of structural unit $i$ as $\delta E_{i}^{u n i t}=\sum_{j} \delta E_{j}^{a t o m}$, where $\delta E_{j}^{a t o m}$ is the contribution of atom $j$ belonging to unit $i$, with the total energy change of atom $j$ shared equally between units to which it belongs. The energy due to one surface atom adjacent to the first crack-tip trailing unit must also be added to the energy of that first unit (see Figure 9c). The slip energy per unit sliding area is then obtained as by dividing by the area per structural unit in the [112]x[110] slip plane, $\delta E_{i}^{\text {unit } /}$ $\left(\sqrt{3} a_{0} / 4\right)$.

The energies to shear each structural unit along the twinning and trailing slip planes, denoted as $\Psi_{i}^{\text {twin }}$ and $\left.\Psi_{i}^{\text {trail }}(i=1, \ldots, n)\right)$, are shown in Figure 10 . Both $\Psi_{1}^{\text {twin }}(\Delta)$ and $\Psi_{1}^{\text {trail }}(\Delta)$ are notably different than all other units, $\Psi_{i \geq 2}^{\text {twin }}(\Delta)$ and $\Psi_{i \geq 2}^{\text {trail }}(\Delta)$. Furthermore, all other units exhibit essentially the bulk response, $\Psi_{i \geq 2}^{\text {twin }}(\Delta) \approx \Psi_{g t f}^{\text {twin }}(\Delta)$ and $\Psi_{i \geq 2}^{\text {trail }}(\Delta) \approx \Psi_{g s f}^{\text {trail }}(\Delta)$. The $\Psi_{2}^{\text {trail }}(\Delta)$ is slightly different than the $\Psi_{g s f}^{\text {trail }}(\Delta)$ presented in Figure 2 because there are small additional contributions to $\Psi_{g s f}^{\text {trail }}(\Delta)$ from the next two planes of atoms just above/below the two 
This is a post-print of the following article: Andric, Predrag; Curtin, W.A. Journal of the Mechanics and Physics of Solids 2018,, 144-161.. The formal publication is available at http://dx.doi.org/10.1016/j.jmps.2018.01.016 @ 2018. This manuscript version is made available under the CC-BY-NC-ND 4.0 license http://creativecommons.org/licenses/by-nc-nd/4.0/

planes of atoms immediately adjacent to the slip plane. Thus, the energy change along the slip plane is in fact nearly $\Psi_{g s f}^{\text {trail }}(\Delta)$ but this is slightly underestimated by only considering the energy change $\Psi_{2}^{\text {trail }}(\Delta)$ of the atoms in the as-defined second structural unit; this difference has no effect on our main results. Several important features emerge from Figure 10. First, the energy of the first structural unit for the twinning process is much smaller than for the bulk twinning process, $\Psi_{1}^{\text {twin }}(\Delta) \ll \Psi_{g t f}^{\text {twin }}(\Delta)$. Thus, during twin partial emission, there is no creation of the step and in

365 fact a decrease in the local surface energy due to atomic rearrangements. The first twinning unit is therefore very easily sheared. Emission of the twin partial requires, however, continued shearing further along the twin plane, and this shearing is controlled by $\Psi_{i \geq 2}^{t w i n}(\Delta) \approx \Psi_{g t f}^{t w i n}(\Delta)$. Second, the energy for slip of the first trailing unit is always accompanied by additional step energy and so, as for the first partial, is far above the bulk GSF energy, $\Psi_{1}^{\text {trail }}(\Delta) \gg \Psi_{g s f}^{\text {trail }}(\Delta)$. The step energy for 370 the trailing partial is smaller than that for the first partial (see Figure 6) because the trailing partial has a screw component along the crack front that does not contribute to step formation (see also Schoeck [7]), but it remains significant and cannot be neglected. Note that zero energy for these cases corresponds to the crystal with the stacking fault (after emission of the first partial dislocation) and so, since the trailing dislocation annihilates the stacking fault, the final energies are lowered and $\Psi_{g s f}^{\text {trail }}\left(\Delta=b_{p}\right)=-\gamma_{s s f}$. Due to the smaller step energy and $\Psi_{g s f}^{\text {trail }}<0$ for $\Delta>$ $3 b_{p} / 4, \Psi_{1}^{\text {trail }}(\Delta)$ does have a maximum value but well above the $\gamma_{u s f}$. Third, the final value of $\Psi_{1}^{\text {trail }}\left(\Delta=b_{p}\right)$, denoted as $\gamma_{\text {step }}^{\text {trail }}$, is $\gamma_{\text {step }}^{\text {trail }} \approx(0.35 \pm 0.03) \gamma_{s}$ for all materials tested; this value is one-half the step energy for first partial emission $\gamma_{\text {step }}^{\text {first }} \approx 0.7 \gamma_{s}$. All the above properties are shown for the particular case of $\mathrm{Ni}$ in Figure 10 but are observed in all other materials studied here.

The above observations reveal the mechanisms for the second partial emission, as follows. Emission of the twinning partial dislocation is controlled not by the very soft first twin unit but by the second twinning unit, which responds like the bulk $\Psi_{g t f}^{t w i n}(\Delta)$ with no step energy. Thus, the Tadmor-Hai theory can be applied for predicting $K_{I e}^{t w i n}$ but with a modification because the second crack tip unit is not at the crack tip. Second, emission of the trailing partial dislocation is similar to emission of the first partial, being controlled by the mechanical instability at high step energies and reducing to the Rice criterion for low step energies. These mechanisms then motivate a new theory for twin emission and use of the Andric-Curtin theory for trailing emission, as discussed below. 
This is a post-print of the following article: Andric, Predrag; Curtin, W.A. Journal of the Mechanics and Physics of Solids 2018,, 144-161.. The formal publication is available at http://dx.doi.org/10.1016/j.jmps.2018.01.016 @ 2018. This manuscript version is made available under the CC-BY-NC-ND 4.0 license http://creativecommons.org/licenses/by-nc-nd/4.0/

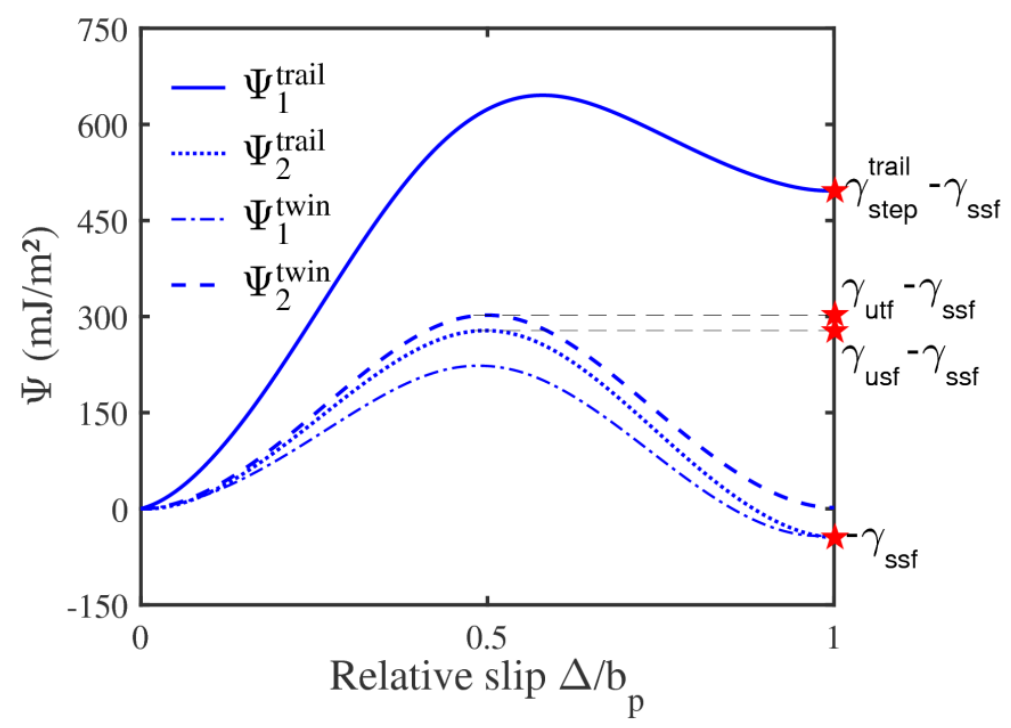

Figure 10: Slip energy change (per unit area) vs. shear displacement of the first trailing unit $\Psi_{1}^{\text {trail }}(\Delta)$ (solid line), first twinning unit $\Psi_{1}^{\text {twin }}(\Delta)$ (dashed-dotted line), second twinning unit $\Psi_{2}^{\text {twin }}(\Delta)$ (dashed line), and second trailing unit $\Psi_{2}^{\text {trail }}(\Delta)$ (dotted line), computed for fcc Ni [18]. The second twinning unit and second trailing unit are negligibly different from the bulk GSF and GTF energies.

\subsection{New theory for crack-tip twinning}

The energy analysis in the previous section shows that it is the second twinning unit that controls the emission of the twinning partial dislocation. However, this key unit is not at the crack tip, and so the Tadmor-Hai theory cannot be applied directly. The Tadmor-Hai theory assumes that the driving force for emission is $K_{I}$, just as for the first partial and trailing partial, and neglects the fact that the twinning plane does not intersect the crack tip. In fact, the stresses acting on the twinning plane differ from those acting on the stacking fault plane, and this difference must be incorporated into the theory.

Specifically, to use the Tadmor and Hai model to predict $K_{I e, t i p}^{\text {twin }}$ we must relate the far-field applied $K_{I}$ to the local stress acting on the second twinning unit. Assuming linear elasticity, at any applied $K_{I}$ the ratio of the shear displacement on the trailing unit (at the crack tip) to the shear displacement on the second twinning unit is some constant that depends only on the elastic constants $C_{i j k l}$,

$$
\frac{\Delta_{1}^{\text {trail }}}{\Delta_{2}^{\text {twin }}}=f^{(1)}\left(C_{i j k l}\right)
$$

For all the fcc materials studied here, and $\theta=70.5^{\circ}$, we find $f^{(1)}\left(C_{i j k l}\right)=1.3-1.42$ where the superscript "1" denotes the first twinning emission (see below). For other crystal orientations we find that the parameter $f^{(1)}\left(C_{i j k l}\right)$ varies only slightly, as shown for Ni in Appendix B. Therefore, to achieve the necessary critical displacement $\Delta_{2, c}^{\text {twin }}$ for twinning at the second structural unit requires an applied stress intensity that is a factor $f^{(1)}\left(C_{i j k l}\right)$ larger than the stress intensity required to attain the same displacement at the crack tip. The critical stress intensity for twinning at the 
This is a post-print of the following article: Andric, Predrag; Curtin, W.A. Journal of the Mechanics and Physics of Solids 2018,, 144-161.. The formal publication is available at http://dx.doi.org/10.1016/j.jmps.2018.01.016 @ 2018. This manuscript version is made available under the CC-BY-NC-ND 4.0 license http://creativecommons.org/licenses/by-nc-nd/4.0/

second structural unit is thus larger than that predicted by Tadmor and Hai by the factor $f^{(1)}\left(C_{i j k l}\right)$. The new criterion for the applied stress intensity for twinning $K_{I e, t i p}^{t w i n}$ is then simply

$$
K_{\text {Ie,tip }}^{\text {twin }}=f^{(1)}\left(C_{i j k l}\right) \sqrt{\left(\gamma_{u t f}-\gamma_{s s f}\right) o\left(\theta, \varphi_{\text {first }}\right)} / F_{12}(\theta) .
$$

410 Figure $3 \mathrm{~b}$ shows the predictions of Eq. 17 for all materials exhibiting "back" twinning, and excellent quantitative agreement is found. The accuracy of the new theory for different slip plane inclination angles is further examined in Appendix B; very good agreement is again obtained.

The above analysis neglects the shielding effect due to the first partial dislocation emission. The shielding by the first partial negative contribution (shielding) adds to the applied stress intensity 415 to give the total crack tip stress intensity. The above crack tip analysis remains valid for controlling the emission process. Therefore, in the presence of the first partial, the remote applied stress intensity factor for "back" twinning is

$$
K_{I e}^{t w i n}=f^{(1)}\left(C_{i j k l}\right)\left(\sqrt{\left(\gamma_{u t f}-\gamma_{s s f}\right) o\left(\theta, \varphi_{f i r s t}\right)} / F_{12}(\theta)+k_{I}^{d}+\frac{F_{22}(\theta)}{F_{12}(\theta)} k_{I I}^{d}\right) .
$$

We have simulated the emission of the second partial dislocation in the presence of the first partial dislocation. The position of the first partial as a function of the applied stress intensity is an outcome of the simulation. Figure 11 shows the predictions of Eq. 18 against the simulation results for all materials showing "back" twinning, and excellent agreement is again obtained. Note that there is no result for Al E [27], because this potential switches from "back" to "forward" twinning in the presence of the first partial dislocation. A possible reason for this change is discussed in Section 4. The results in Figure 11 reaffirm the accuracy of the detailed local crack-tip analysis of the twinning process.

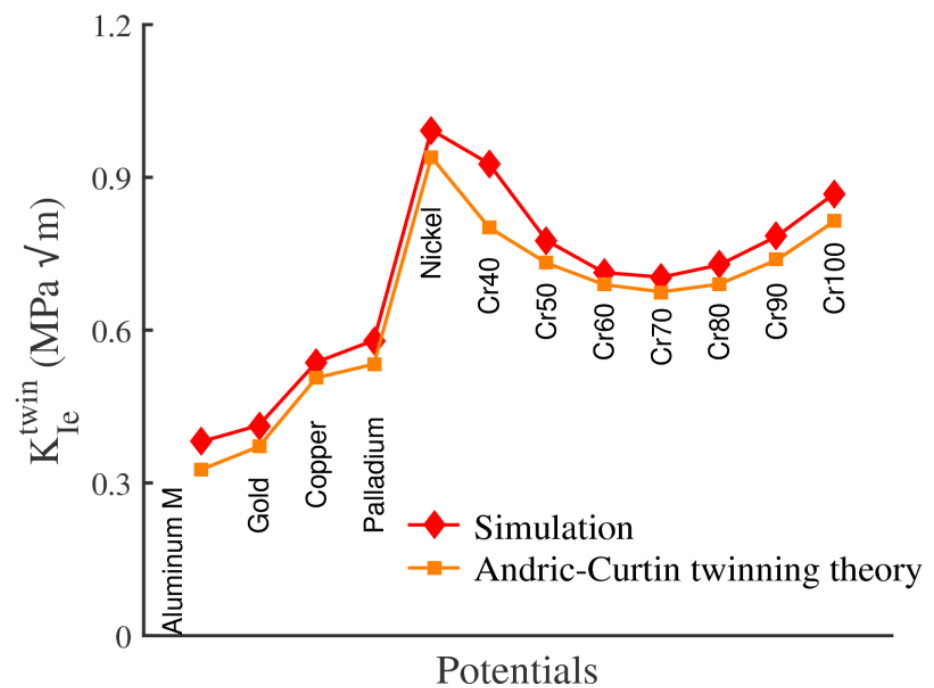

Figure 11: Critical stress intensity factor for the emission of the "back" twinning partial dislocation including the effect of shielding due to the first partial dislocation, as observed in the simulations (red diamonds) and as computed by the new twinning theory (orange squares). 
This is a post-print of the following article: Andric, Predrag; Curtin, W.A. Journal of the Mechanics and Physics of Solids 2018,, 144-161.. The formal publication is available at http://dx.doi.org/10.1016/j.jmps.2018.01.016 @ 2018. This manuscript version is made available under the CC-BY-NC-ND 4.0 license http://creativecommons.org/licenses/by-nc-nd/4.0/

\subsection{Trailing partial emission: why twinning is observed in all simulations}

We now address why simulations show that twinning is always preferred when $\varphi_{\text {first }}=0$. Twinning occurs when $K_{I e}^{\text {twin }}<K_{I e}^{\text {trail }}$. Above we predicted $K_{I e}^{\text {twin }}$ accurately, and therefore we must now assess $K_{I e}^{\text {trail }}$. The energy analysis in Figure 10 shows that the step energy during trailing emission is significant, and this necessitates the application of the Andric-Curtin theory for predicting $K_{I e}^{\text {trail }}$.

We first recall the analytical expression for $G_{I e}^{\text {first }}$ (Eq. 15). Furthermore, the step energy is sufficiently high, $\gamma_{\text {step }}^{\text {trail }} \gg\left(\gamma_{u s f}-\gamma_{s s f}\right)$ (see Table 1$)$, in most materials studied and $\gamma_{\text {step }}^{\text {trail }} \approx$ $435 \gamma_{\text {step }}^{\text {first }} / 2$. We thus substitute this latter expression into Eq. 15 and also replace $\gamma_{u s f}$ by $\left(\gamma_{u s f}-\right.$ $\left.\gamma_{s s f}\right)$ in Eq. 15, leading to

$$
G_{I e}^{\text {trail }}=\left\{\begin{array}{ll}
0.0725 \gamma_{s}+0.5\left(\gamma_{u s f}-\gamma_{s s f}\right), & \gamma_{s}>6,9\left(\gamma_{u s f}-\gamma_{s s f}\right) \\
\left(\gamma_{u s f}-\gamma_{s s f}\right), & \gamma_{s}<6,9\left(\gamma_{u s f}-\gamma_{s s f}\right)
\end{array} .\right.
$$

The critical stress intensity factor for the emission of the trailing partial dislocation $K_{\text {Ie,tip }}^{\text {trail }}$ is then easily computed as

$$
K_{\text {Ie,tip }}^{\text {trail }}=\sqrt{G_{\text {Ie,tip }}^{\text {trail }} o\left(\theta, \varphi_{\text {trail }}\right)} / F_{12}(\theta) \cos \left(\varphi_{\text {trail }}\right) .
$$

Using Eq. 20 to predict $K_{I e, t i p}^{\text {trail }}$, we find $K_{I e, t i p}^{\text {twin }}<\left(K_{I e, t i p}^{\text {trail }}\right)_{N e w}$ for all materials showing "back"

440 twinning, in a perfect agreement with simulations (see Table 1). Note that Eq. 19 also captures the correct limit with respect to the ratio $\gamma_{s} /\left(\gamma_{u s f}-\gamma_{s s f}\right)$, where the Rice theory for $K_{I e}^{\text {trail }}$ is applicable for materials with a low step energy. Since emission of the trailing partial never happens at $\mathrm{T}=0 \mathrm{~K}$, we cannot prove the quantitative accuracy of Eq. 20 but it is consistent with all simulations. As for the analysis of twinning, careful attention to the local crack-tip energetics provides crucial insights for understanding of the mechanics of the crack-tip dislocation emission. 
This is a post-print of the following article: Andric, Predrag; Curtin, W.A. Journal of the Mechanics and Physics of Solids 2018,, 144-161.. The formal publication is available at http://dx.doi.org/10.1016/j.jmps.2018.01.016 @ 2018. This manuscript version is made available under the CC-BY-NC-ND 4.0 license http://creativecommons.org/licenses/by-nc-nd/4.0/

\begin{tabular}{cccc}
\hline Material & $\begin{array}{c}\text { Twinning partial } \\
\mathrm{K}_{\text {Ie,tip }} \\
\left(\mathrm{MPa} \mathrm{m}^{1 / 2}\right)\end{array}$ & $\begin{array}{c}\text { Trailing partial } \\
\mathrm{K}_{\mathrm{Ie}, \mathrm{tip}} \\
\left(\mathrm{MPa} \mathrm{m}^{1 / 2}\right)\end{array}$ & $\begin{array}{c}\text { Ratio } \\
\gamma_{\mathrm{s}}\left(\gamma_{\text {usf }}-\gamma_{\mathrm{ss}}\right)\end{array}$ \\
\hline $\mathrm{Al}$ Mishin & 0.274 & 0.358 & 39.06 \\
$\mathrm{Al}$ Ercolessi & 0.264 & 0.377 & 39.06 \\
$\mathrm{Au}$ & 0.355 & 0.406 & 8.66 \\
$\mathrm{Ag}{ }^{*}$ & 0.382 & 0.474 & 5.29 \\
$\mathrm{Cu}$ & 0.485 & 0.608 & 10.54 \\
$\mathrm{Pd}$ & 0.508 & 0.615 & 10.05 \\
$\mathrm{Ni}{ }^{*}$ & 0.881 & 1.035 & 6.73 \\
$\mathrm{Cr} 10^{*}$ & 1.142 & 1.443 & 3.86 \\
$\mathrm{Cr} 20^{*}$ & 1.002 & 1.240 & 4.69 \\
$\mathrm{Cr} 33^{*}$ & 0.842 & 0.997 & 6.22 \\
$\mathrm{Cr} 40$ & 0.772 & 0.896 & 7.26 \\
$\mathrm{Cr} 50$ & 0.684 & 0.789 & 9.42 \\
$\mathrm{Cr} 60$ & 0.628 & 0.722 & 12.42 \\
$\mathrm{Cr} 70$ & 0.598 & 0.677 & 16.74 \\
$\mathrm{Cr} 80$ & 0.598 & 0.660 & 21.73 \\
$\mathrm{Cr} 90$ & 0.630 & 0.671 & 25.03 \\
$\mathrm{Cr} 100$ & 0.692 & 0.705 & 25.22 \\
\hline
\end{tabular}

Table 1: Predicted critical stress intensities for twinning (Eq. 17) and trailing (Eq. 20) partial dislocation emission, for $17 \mathrm{fcc}$ materials. The ratio of surface energy to unstable minus stable stacking fault energy is shown, and the Rice theory is applicable when this value is below 6.9, as indicated for those materials marked by an asterisk.

\section{Forward twinning and twin thickening in fec metals}

Both the Tadmor-Hai theory and the new theory for crack-tip twinning are appropriate for predicting $K_{I e}^{\text {twin }}$ when "back" twinning occurs. As noted earlier, the process of "forward" twinning can occur by crack advancement by one lattice spacing followed by sliding along the twin plane at the new crack tip (see Figure $5 \mathrm{c}$ ). At $\mathrm{T}=0 \mathrm{~K}$, the crack growth process requires attainment of the Griffith critical stress intensity $K_{I c}$ for cleavage. "Forward" twinning thus occurs for materials where $K_{I c}<K_{I e}^{t w i n}$, with $K_{I e}^{\text {twin }}$ computed via Eq. 17 . That is, before "back" twinning can occur, the crack cleaves and the stress intensity is sufficient to nucleate the twinning dislocation at the new crack tip ahead of the original stacking fault plane. Figure 12a shows that Ag, Cr10 and Cr20 satisfy the criteria for "forward" twinning and these are indeed all cases where forward twinning is observed. The Cr33 material also shows "forward" twinning in simulations even though $K_{I C}>$ $K_{I e}^{\text {twin }}$, but the difference is quite small and discrepancies may arise due to non-linear effects that are absent from our models. The standard Tadmor-Hai is unable to distinguish between "back" and "forward" twinning, and predictions using Eq. 6 yield $K_{I c}>K_{I e}^{\text {twin }}$ for all materials, indicating that 
This is a post-print of the following article: Andric, Predrag; Curtin, W.A. Journal of the Mechanics and Physics of Solids 2018,, 144-161.. The formal publication is available at http://dx.doi.org/10.1016/j.jmps.2018.01.016 @ 2018. This manuscript version is made available under the CC-BY-NC-ND 4.0 license http://creativecommons.org/licenses/by-nc-nd/4.0/

"back" twinning would be expected in all cases. The ability to distinguish between two different twinning modes provides additional support for the qualitative and quantitative accuracy of the analysis and models (Eqs. 17 and 18) presented in this paper.

The recognition of the different processes of "back" and "forward" twinning now changes our understanding of further crack-tip twinning, after the first twin partial emission, as follows. "Back" twinning for the second twin partial, thickening the twin by one additional layer, would occur by nucleation even further from the crack tip than for the first "back" twin nucleation. Being further from the crack tip necessitates a higher applied $K_{I}$ to reach nucleation even though the underlying process remains the same. In other words, the scaling factor $f^{(2)}\left(C_{i j k l}\right)$ for second "back" twin partial emission is larger than the value $f^{(1)}\left(C_{i j k l}\right)=1.3-1.42$ for the first "back" twin partial. Each subsequent "back" twinning partial thus requires further increases in applied $K_{I}$. At some point, the applied $K_{I}$ reaches $K_{I c}$, after which the twin process changes to thickening by the "forward" twinning mechanism. Assuming that the sharp crack cleavage value of $K_{I c}$ applies independent of the evolving shape of the crack tip as the twin thickens, and ignoring for the moment the shielding effects of the twinning dislocations, the "forward" twinning occurs at the $\mathrm{n}^{\text {th }}$ twin emission for which

$$
f^{(n)}\left(C_{i j k l}\right) \sqrt{\left(\gamma_{u t f}-\gamma_{s s f}\right) o\left(\theta, \varphi_{\text {first }}\right)} / F_{12}(\theta)>K_{I c} .
$$

Since the $f^{(n)}$ grow rapidly $\left(f^{(1)} \sim 1.4 ; f^{(2)} \sim 1.7 ; f^{(3)} \sim 2.0\right)$, the onset of "forward" twinning is expected after only a few "back" twinning events. Figure $12 \mathrm{~b}$ shows the Griffith value for cleavage 480 along with the simulation stress intensity factor at which "forward" twinning occurs, without shielding effects due to previously-emitted partial dislocations. The onset of "forward" twinning occurs at an applied $K_{I}$ very close to the Griffith cleavage $K_{I c}$. The only exception is the $\operatorname{Cr} 10$ material where "forward" twinning occurs high above $K_{I c}$; this is due to some rearrangement of crack-tip atoms that delays "forward" twinning emission although the cleavage process starts 485 developing at $K_{I}$ very close to $K_{I c}$. Figure $12 \mathrm{~b}$ also indicates the number of "back" twinning partial dislocations prior to "forward" twinning as observed in the simulations and as estimated by Eq. 21 (given in parentheses). The predictions are in a very good agreement with the simulations for most of the materials studied here. The number of "back" twinning partials observed in the Cr70-Cr100 materials is larger than estimated via Eq. 21; this discrepancy may arise because of changes to the 490 crack tip geometry being more important for these materials. These results confirm that our analysis captures the change from "back" to "forward" twinning with good accuracy. 
This is a post-print of the following article: Andric, Predrag; Curtin, W.A. Journal of the Mechanics and Physics of Solids 2018,, 144-161.. The formal publication is available at http://dx.doi.org/10.1016/j.jmps.2018.01.016 @ 2018. This manuscript version is made available under the CC-BY-NC-ND 4.0 license http://creativecommons.org/licenses/by-nc-nd/4.0/

As noted earlier, previously-emitted partial dislocations act to shield the crack tip. This shielding occurs for both emission and cleavage processes. Ignoring mix-mode cleavage, the onset of "forward" twinning can be estimated to occur at the $\mathrm{n}^{\text {th }}$ twinning event, where $\mathrm{n}$ satisfies

$$
f^{(n)}\left(C_{i j k l}\right)\left[\sqrt{\left(\gamma_{u t f}-\gamma_{s s f}\right) o\left(\theta, \varphi_{f i r s t}\right)} / F_{12}(\theta)+\sum_{i=1}^{n}\left(k_{I}^{d}+\frac{F_{22}(\theta)}{F_{12}(\theta)} k_{I I}^{d}\right)\right]>K_{I C}+\sum_{i=1}^{n} k_{I}^{d}
$$

495 for any twin dislocation array, $k_{I I}^{d} \propto k_{I}^{d}$ while $f^{(n)}\left(C_{i j k l}\right)>1$. Therefore, the onset of "forward" twinning should occur sooner (smaller $n$ ) in the presence of the shielding that exists in the real situation.
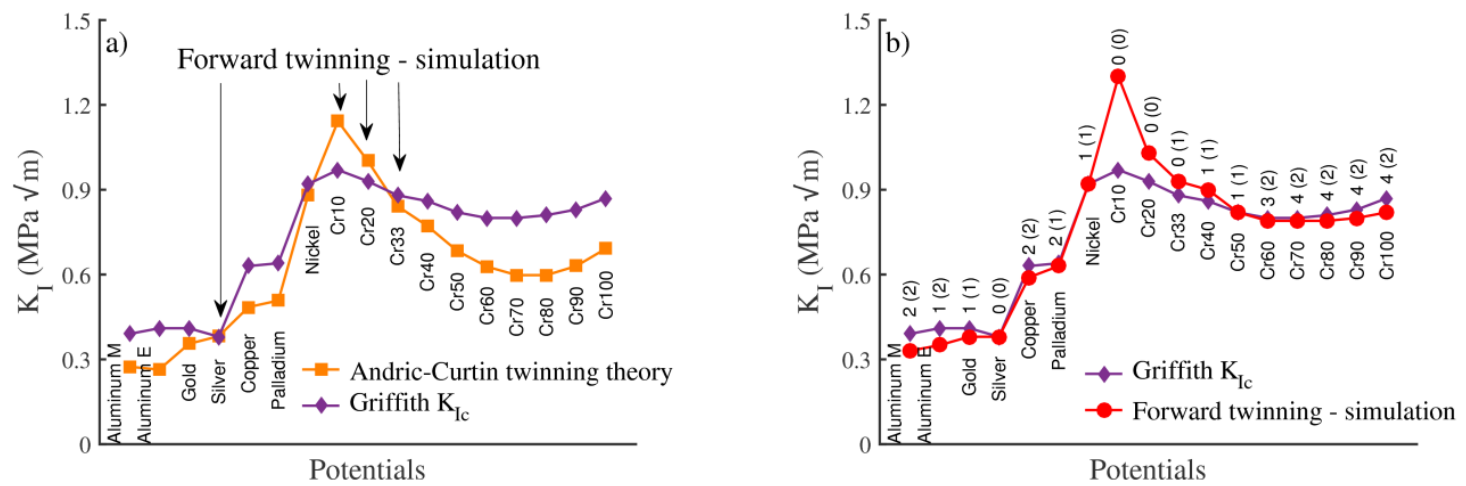

Figure 12: a) Critical stress intensity factor for Griffith cleavage $K_{I c}$ (purple diamonds) and predicted "back" twinning partial emission $K_{I e, t i p}^{\text {twin }}$ (orange squares); forward emission is predicted when $K_{I c}<$ $K_{I e}^{\text {twin }}$ and the cases where forward twining is observed in simulations are indicated. b) Critical stress intensity factor for Griffith cleavage $K_{I C}$ (purple diamonds) and simulated value of the critical stress intensity at the onset of "forward" twinning (red circles), showing that forward twinning starts at $K_{I C}$. The number of "back" twinning events prior to "forward" twinning is indicated for each material, as observed in simulation and, in parentheses, as predicted by Eq. 21

After the cleavage event, the "forward" twinning process is similar to the first emission process, involving step creation. The "forward" twinning should be slightly easier, $K_{I e}^{\text {forward }}<$ $K_{I e}^{f i r s t}$, since the process eliminates the stacking fault in favour of the twinning faults rather than creating a stacking fault. Therefore, since the first partial emission necessarily satisfies $K_{I e}^{\text {first }}<$ $K_{I c}$, the entire twinning process is ultimately controlled by the conditions established for first partial emission. The brittle/quasi-brittle transition is then truly determined by the conditions for the first partial emission, even though increasing applied stress intensity factors are required to drive subsequent twinning! And, the quasi-brittle domain actually intimately involves the cleavage process - the crack begins to cleave but then is able to immediately emit another twinning partial dislocation, leading to additional shielding.

Finally, crack tip cleavage may require local crack tip stress intensities that differ slightly from $K_{I c}$. Cleavage above $K_{I c}$ at $\mathrm{T}=0 \mathrm{~K}$ can occur due to lattice trapping [22]. Probably of more importance is that, as twinning (or trailing partial) emission evolve, the shape of the crack tip changes and this changes the stresses acting around the crack tip. The influence of the crack shape 
This is a post-print of the following article: Andric, Predrag; Curtin, W.A. Journal of the Mechanics and Physics of Solids 2018,, 144-161.. The formal publication is available at http://dx.doi.org/10.1016/j.jmps.2018.01.016 @ 2018. This manuscript version is made available under the CC-BY-NC-ND 4.0 license http://creativecommons.org/licenses/by-nc-nd/4.0/

on $K_{I c}$ at the atomic scale was first analysed by Gumbsch [33]. The twinning process leads to the development of a wedge shape, which then involves stress concentrations at the two corners and a

515 differently-oriented crystal in the twinned region. If the cleavage process requires local stresses different than those for the sharp crack, then this factor must be embedded into an effective value of $K_{I C}$ to be used in the analyses discussed here.

\section{Concluding remarks}

The continuum Rice and the Tadmor-Hai theories for predicting the critical stress intensity factor for crack tip dislocation emission are well-accepted and widely used in solid-mechanics community. However, atomistic simulations on a wide range of fcc metals have revealed that their predictions are not highly accurate. We previously demonstrated that Rice theory is not accurate in predicting the critical stress intensity factor for the emission of the first partial dislocation $K_{I e}^{\text {first }}$. Here, we have also shown that the Tadmor-Hai theory is even less accurate in predicting the critical stress intensity factor for twinning partial dislocation emission $K_{I e, t i p}^{t w i n}$.

A discrete analysis of the local energetics and deformation in the crack tip vicinity is shown to be the key to the development of a predictive theory for $K_{I e}$. Our previous analysis revealed that the emission of the first partial dislocation is always accompanied by the surface step creation, which is neglected in the Rice theory. The surface step creation changes the emission from being energy-controlled to being controlled by a mechanical instability at the crack tip, and a new theory for the leading partial emission $K_{I e}$ was shown to be in excellent agreement with the simulation results. Here, a similar discrete energetic approach reveals that the emission of the twinning partial dislocation does not involve step creation and, in fact, there is a decrease in energy near the crack tip. Twinning is then controlled by the second twinning unit along the twinning plane, which follows the bulk GTF energy. However, the stress acting at the second twinning unit is lower than that at the crack tip. Accounting for this lower stress leads to predictions for the $K_{I e}$ in very good agreement with simulations. Additional analyses demonstrate why twinning is always observed at $\mathrm{T}=0 \mathrm{~K}$ in all materials studied here. Finally, we find a new mode of "forward" twinning, which starts

540 after only a few (0-4) "back" emission events and is responsible for further twin thickening. "Forward" twinning is closely connected to Griffith cleavage, and our analysis demonstrates that the twinning versus cleavage competition is then fully determined by the competition for the very first partial dislocation emission.

The analysis here has been at $\mathrm{T}=0 \mathrm{~K}$. The competition between trailing and twinning 545 emission at finite $\mathrm{T}$ has been shown to be a competition between $K$-dependent energy barriers for the two processes [34]. While the energy barriers computed according to the Rice and Tadmor-Hai 
This is a post-print of the following article: Andric, Predrag; Curtin, W.A. Journal of the Mechanics and Physics of Solids 2018,, 144-161.. The formal publication is available at http://dx.doi.org/10.1016/j.jmps.2018.01.016 @ 2018. This manuscript version is made available under the CC-BY-NC-ND 4.0 license http://creativecommons.org/licenses/by-nc-nd/4.0/

theories show a transition from twinning to trailing emission at some time and temperature, the results are not completely consistent with simulations [16, 34]. The present theory, quantitatively explaining the competition at $\mathrm{T}=0 \mathrm{~K}$, provides the necessary basis for future analysis of the energy barriers and competition at finite temperatures. We will report on efforts in this direction in the future.

Acknowledgements: This work was supported by the European Research Council through the Advanced Grant "Predictive Computational Metallurgy", ERC Grant agreement No.339081 PreCoMet.

\section{Appendix A. Cracks in homogeneous anisotropic elastic media}

Consider an infinite anisotropic elastic body having a traction free crack of a length $2 a$, located at $y=0$, $|x|<a$. The body is subjected to an uniform stress applied at infinity $\sigma_{i j}^{\infty},(i, j=x, y)$. By using the superposition principle, the solution to this problem can be found by summing: (i) the solution for a non-cracked body subjected to a uniform stress in infinity $\sigma_{i j}^{\infty}$ and (ii) the solution for a crack body with crack surfaces subjected to uniform tractions $t_{\Gamma}$ and $-t_{\Gamma}$ (positive for upper and negative for lower crack surface) with the stress vanishing in infinity. Within the boundary conditions mentioned above, the displacement field, and the stress function from which the stress field can be found, are given by the following equations:

$$
\begin{aligned}
& u=\operatorname{Re}\left\{A\left\langle f_{0}\left(z_{\alpha}\right)\right\rangle B^{-1}\right\} t_{\Gamma}, \\
& \psi=\operatorname{Re}\left\{B\left\langle f_{0}\left(z_{\alpha}\right)\right\rangle B^{-1}\right\} t_{\Gamma} .
\end{aligned}
$$

where

$\left\langle f_{0}\left(z_{\alpha}\right)\right\rangle=\operatorname{diag}\left[f\left(z_{1}\right), f\left(z_{2}\right), f\left(z_{3}\right)\right]$,

$f(z)=\sqrt{z^{2}-a^{2}}-z, \quad z=x+p y$.

$\mathrm{A}$ and $\mathrm{B}$ are the $3 \times 3$ normalized complex matrices, while $p$ is one of the eigenvalues defined in the Stroh formalism. More details on finding A, B and $p$ is given in the following text. In our problem of interest it is more convenient to use the polar coordinates with the origin at the crack tip where $z=r(\cos \theta+p \sin \theta)+a$, and the asymptotic solution for the elastic field when $r \rightarrow 0$ leading to

$$
u=\sqrt{2 \operatorname{ar}} \operatorname{Re}\left\{A\left\langle\sqrt{\cos \theta+p_{\alpha} \sin \theta}\right\rangle B^{-1}\right\} t_{\Gamma}
$$

for displacement field, and

$$
\begin{aligned}
& t_{1}=-\sqrt{\frac{a}{2 r}} \operatorname{Re}\left\{B\left(\sqrt{\frac{p_{\alpha}}{\cos \theta+p_{\alpha} \sin \theta}}\right) B^{-1}\right\} t_{\Gamma} \\
& t_{2}=-\sqrt{\frac{a}{2 r}} \operatorname{Re}\left\{B\left(\sqrt{\frac{1}{\cos \theta+p_{\alpha} \sin \theta}}\right) B^{-1}\right\} t_{\Gamma}
\end{aligned}
$$

for the stress field, where $t_{1}=\left[\sigma_{x x}, \sigma_{y x}, \sigma_{z x}\right]^{T}$ and $t_{2}=\left[\sigma_{x y}, \sigma_{y y}, \sigma_{z y}\right]^{T}$. The stress intensity factor at the tip is then $K=\left[K_{I I}, K_{I}, K_{I I I}\right]^{T}=\sqrt{\pi a} t_{\Gamma}$. 
This is a post-print of the following article: Andric, Predrag; Curtin, W.A. Journal of the Mechanics and Physics of Solids 2018,, 144-161.. The formal publication is available at http://dx.doi.org/10.1016/j.jmps.2018.01.016 @ 2018. This manuscript version is made available under the CC-BY-NC-ND 4.0 license http://creativecommons.org/licenses/by-nc-nd/4.0/

For an atomically sharp, semi-infinite crack it can be shown that far-field energy release rate is

$$
G=K^{T} \frac{1}{2} L^{-1} K
$$

where

$$
L^{-1}=\operatorname{Re}\left(i A B^{-1}\right) .
$$

The complex matrices $\mathrm{A}$ and $\mathrm{B}$, and the eigenvalue $p$ can be found by solving the eigenvalue problem given by the following equations

$$
\begin{aligned}
& N \xi=p \xi \\
& N=\left[\begin{array}{ll}
N_{1} & N_{2} \\
N_{3} & N_{1}^{T}
\end{array}\right], \xi=\left[\begin{array}{l}
A \\
B
\end{array}\right]
\end{aligned}
$$

with

$$
N_{1}=-T^{-1} R^{T}, N_{2}=T^{-1}, N_{3}=R T^{-1} R^{T}-Q
$$

and

$$
Q_{i k}=C_{i 1 k 1}, R_{i k}=C_{i 1 k 2,} T_{i k}=C_{i 2 k 2} .
$$

In the last equation $C_{i j k l}$ is a material stiffness tensor.

Under pure Mode I loading $K=\left[0, K_{I}, 0\right]^{T}$, the critical stress intensity for Griffith cleavage is

$$
K_{I c}=\sqrt{2 \gamma_{s} \Lambda_{22}^{-1}}
$$

with

$$
\Lambda=\frac{1}{2} L^{-1},
$$

while the critical stress intensity factor for dislocation emission when the slip plane is inclined to an angle $\theta$ is

$$
K_{I e}=\sqrt{G_{I e} O(\theta, \varphi)} / F_{12}(\theta) .
$$

In the last equation $G_{I e}$ is the critical energy release rate for crack tip dislocation emission computed according to Andric-Curtin theory, while $o(\theta, \varphi)$ represents the material elastic property for shear along the slip direction given by

$$
o(\varphi, \theta)=s_{i}(\varphi) \Lambda_{i j}^{(\theta)^{-1}} s_{j}(\varphi)
$$

with $s(\varphi)$ being the slip vector in the constrained path approximation and

$$
\Lambda_{i j}^{(\theta)}=\Omega_{i k} \Lambda_{k l} \Omega_{l j} .
$$

585 In the last equation $\Omega$ is the rotation matrix given by

$$
\Omega=\left[\begin{array}{ccc}
\cos \theta & \sin \theta & 0 \\
-\sin \theta & \cos \theta & 0 \\
0 & 0 & 1
\end{array}\right] .
$$

For more details the reader is referred to [20,21, 35]. Table 2 shows material properties used for predicting the competition between trailing vs. twinning partial emission in all materials studied in this work. 
This is a post-print of the following article: Andric, Predrag; Curtin, W.A. Journal of the Mechanics and Physics of Solids 2018,, 144-161.. The formal publication is available at http://dx.doi.org/10.1016/j.jmps.2018.01.016 @ 2018. This manuscript version is made available under the CC-BY-NC-ND 4.0 license http://creativecommons.org/licenses/by-nc-nd/4.0/

\begin{tabular}{|c|c|c|c|c|c|c|c|c|c|c|c|c|c|}
\hline Material & $(\AA)$ & GPa & $\mathrm{C}_{12}$ & $\mathrm{C}_{44}$ & $\begin{array}{c}\mathrm{o}\left(\theta=70^{\circ},\right. \\
\varphi=0) \\
\mathrm{GPa}\end{array}$ & $\begin{array}{c}\mathrm{o}\left(\theta=70^{\circ},\right. \\
\left.\varphi=60^{\circ}\right) \\
\mathrm{GPa}\end{array}$ & $\Lambda_{22}$ & $(\mathrm{~J} / \mathrm{m} 2)$ & $(\mathrm{J} / \mathrm{m} 2)$ & $(\mathrm{J} / \mathrm{m} 2)$ & $f^{(1)}$ & $\mathrm{f}^{(2)}$ & $\begin{array}{c}\gamma_{\mathrm{s}}\left(\mathrm{J} / \mathrm{m}^{2}\right) \\
(111) \\
(\mathrm{J} / \mathrm{m} 2)\end{array}$ \\
\hline $\mathrm{Al} \mathrm{M}$ [18] & 4.050 & 113.4 & 61.5 & 31.6 & 87.9 & 64.9 & 88.7 & 0.167 & 0.145 & 0.220 & 1.314 & 1.646 & 0.871 \\
\hline $\mathrm{AlE}$ [27] & 4.032 & 118.0 & 62.2 & 36.7 & 97.0 & 72.3 & 98.2 & 0.119 & 0.106 & 0.168 & 1.313 & 1.666 & 0.871 \\
\hline $\mathrm{Au}[29]$ & 4.080 & 183.2 & 158.7 & 45.3 & 101.0 & 60.6 & 103.7 & 0.097 & 0.005 & 0.099 & 1.422 & 1.819 & 0.796 \\
\hline $\mathrm{Ag}$ [29] & 4.090 & 129.1 & 91.7 & 56.7 & 115.4 & 78.1 & 120.0 & 0.119 & 0.002 & 0.119 & 1.349 & 1.713 & 0.619 \\
\hline $\mathrm{Cu}[28]$ & 3.615 & 169.9 & 122.6 & 76.2 & 152.1 & 101.7 & 158.4 & 0.162 & 0.044 & 0.184 & 1.350 & 1.729 & 1.240 \\
\hline $\operatorname{Pd}[29]$ & 3.890 & 221.1 & 183.0 & 72.6 & 152.2 & 93.8 & 157.4 & 0.145 & 0.015 & 0.152 & 1.400 & 1.796 & 1.301 \\
\hline $\mathrm{Ni}[18]$ & 3.520 & 247.9 & 147.8 & 124.8 & 249.8 & 181.0 & 260.0 & 0.368 & 0.125 & 0.428 & 1.299 & 1.654 & 1.631 \\
\hline $\operatorname{Cr} 10$ [30] & 3.497 & 301.3 & 171.5 & 156.7 & 311.2 & 229.1 & 324.1 & 0.425 & 0.051 & 0.451 & 1.314 & 1.669 & 1.445 \\
\hline $\mathrm{Cr} 20$ [30] & 3.507 & 276.6 & 165.6 & 148.7 & 287.5 & 208.2 & 300.4 & 0.356 & 0.049 & 0.380 & 1.324 & 1.681 & 1.441 \\
\hline Cr33 [30] & 3.522 & 246.6 & 158.1 & 138.5 & 257.8 & 181.9 & 270.5 & 0.288 & 0.057 & 0.317 & 1.338 & 1.699 & 1.434 \\
\hline Cr40 [30] & 3.529 & 232.6 & 154.7 & 133.7 & 243.7 & 169.2 & 256.3 & 0.264 & 0.067 & 0.298 & 1.345 & 1.705 & 1.430 \\
\hline Cr50 [30] & 3.541 & 213.1 & 150.8 & 127.6 & 223.7 & 150.5 & 236.2 & 0.240 & 0.089 & 0.285 & 1.358 & 1.726 & 1.424 \\
\hline Cr60 [30] & 3.552 & 205.5 & 150.3 & 124.6 & 214.7 & 141.7 & 227.1 & 0.232 & 0.117 & 0.289 & 1.367 & 1.738 & 1.418 \\
\hline Cr70 [30] & 3.563 & 204.5 & 152.8 & 124.6 & 212.4 & 138.2 & 224.8 & 0.237 & 0.152 & 0.308 & 1.375 & 1.747 & 1.413 \\
\hline Cr80 [30] & 3.572 & 211.2 & 159.0 & 127.9 & 218.1 & 141.2 & 230.8 & 0.255 & 0.190 & 0.340 & 1.383 & 1.751 & 1.408 \\
\hline Cr90 [30] & 3.579 & 225.6 & 168.9 & 139.4 & 231.8 & 150.7 & 245.2 & 0.287 & 0.231 & 0.385 & 1.390 & 1.751 & 1.404 \\
\hline $\mathrm{Cr} 100$ [30] & 3.584 & 247.3 & 182.5 & 145.5 & 253.3 & 166.3 & 267.5 & 0.328 & 0.272 & 0.441 & 1.396 & 1.727 & 1.400 \\
\hline
\end{tabular}

Table 2: Material properties used for predicting the trailing vs. twinning partial emission in all materials studied in this work.

\section{Appendix B. Assessment of the theory for different slip plane orientations}

The new theory is shown to be in excellent agreement with simulations for the slip plane inclination angle $\theta=70.5^{\circ}$ in different fcc metals. We now examine the accuracy of the theory using molecular statics simulations for $\theta=35.3^{\circ}, 54.7^{\circ}, 70.5^{\circ}$ and $90^{\circ}$ in the specific case of fcc $\mathrm{Ni}$ [18]. We perform standard K-test without the shielding effect, as described above.

First, Table 3 shows that the parameter $f^{(1)}\left(C_{i j k l}\right)$ changes very little due to anisotropy and a slightly different position of the twinning unit with respect to the trailing unit. Second, in every case we observe the twinning partial emission, as expected. We then compare the critical stress intensity factors $K_{\text {Ie,tip }}^{\text {twin }}$ observed in the simulations with the theoretical predictions. We find excellent quantitative and qualitative agreement, except at $\theta=35.3^{\circ}$. In this case the new theory is notable higher, despite the fact that we accurately predict the twinning mode (see Table 3 ). This discrepancy arises by the presence of the two active symmetrical slip systems that interact slightly. We found similar behaviour in the case of the first partial dislocation emission. More explanations on this issue can be found in [15].

For other crack orientations, especially when $\varphi_{\text {first }} \neq 0$, the theory applies when using the correct component(s) of the Burgers vector(s) that couple to the applied crack tip stress field. Examining all possible cases is far beyond the scope of the present paper. To conclude, we believe that the work presented here, being an entirely new type of analysis for a long-standing problem, is sufficient for validating the new theory. 
This is a post-print of the following article: Andric, Predrag; Curtin, W.A. Journal of the Mechanics and Physics of Solids 2018,, 144-161.. The formal publication is available at http://dx.doi.org/10.1016/j.jmps.2018.01.016 @ 2018. This manuscript version is made available under the CC-BY-NC-ND 4.0 license http://creativecommons.org/licenses/by-nc-nd/4.0/

\begin{tabular}{cccccccccc}
\hline$\theta\left(^{\circ}\right)$ & $\mathrm{X}$ & $\mathrm{Y}$ & $\mathrm{Z}$ & $f^{(1)}$ & $\begin{array}{c}K_{\text {Ie,new }}^{\text {twin }} \\
(\mathrm{MPa} \sqrt{\mathrm{m}})\end{array}$ & $\begin{array}{c}K_{\text {Ie,sim }}^{\text {twin }} \\
(\mathrm{MPa} \sqrt{\mathrm{m}})\end{array}$ & $\begin{array}{c}K_{I C} \\
(\mathrm{MPa} \sqrt{\mathrm{m}})\end{array}$ & $\begin{array}{c}\text { Predicted } \\
\text { mode }\end{array}$ & $\begin{array}{c}\text { Simulation } \\
\text { mode }\end{array}$ \\
\hline 35.3 & {$[001]$} & {$[110]$} & {$[\overline{1} 10]$} & 1.22 & 1.16 & 0.92 & 1.06 & forward & forward \\
54.7 & {$[\overline{1} \overline{1} 4]$} & {$[221]$} & {$[\overline{1} 10]$} & 1.29 & 0.93 & 0.98 & 1.01 & back & back \\
70.5 & {$[\overline{1} \overline{1} 12]$} & {$[111]$} & {$[\overline{1} 10]$} & 1.30 & 0.88 & 0.89 & 0.92 & back & back \\
90.0 & {$[\overline{1} \overline{1} 1]$} & {$[112]$} & {$[\overline{1} 10]$} & 1.33 & 0.99 & 0.93 & 0.99 & forward & forward \\
\hline
\end{tabular}

Table 3: Critical stress intensity factor for twinning partial dislocation emission for different slip plane inclination angles $\theta$ as predicted by the Andric-Curtin twinning theory and as observed in the simulations. The observed and predicted twinning mode is shown, along with the Griffith $K_{I C}$ and the parameter $f^{(1)}\left(C_{i j k l}\right)$.

\section{References}

615 [1] Griffith AA. The phenomena of rupture and flow in solids. Philosophical transactions of the royal society of London. Series A, containing papers of a mathematical or physical character. 1921Jan 1;221:163-98.

[2] Warner DH, Curtin WA, Qu S. Rate dependence of crack-tip processes predicts twinning trends in fcc metals. Nature materials. 2007 Nov 1;6(11):876.

[3] Argon AS. Britle to ductile transition in cleavage fracture. Acta Metallurgica. 1987 Jan 1;35(1):185-96.

620 [4] Armstrong RW. Cleavage crack propagation within crystals by the Griffith mechanism versus a dislocation mechanism. Materials science and engineering. 1966 Nov 1;1(4):251-4.

[5] Kelly A, Tyson WR, Cottrell AH. Ductile and brittle crystals. Philosophical magazine. 1967 Mar 1;15(135):567-86.

[6] Rice JR, Thomson R. Ductile versus brittle behavior of crystals. Philosophical magazine. 1974 Jan 1;29(1):7397.

[7] Schoeck G. The emission of dislocations from crack tips: A critical assessment. Materials Science and Engineering: A. 2003 Sep 15;356(1):93-101.

[8] Rice JR. Dislocation nucleation from a crack tip: an analysis based on the Peierls concept. Journal of the Mechanics and Physics of Solids. 1992 Dec 31;40(2):239-71.

630 [9] Hai S, Tadmor EB. Deformation twinning at aluminum crack tips. Acta Materialia. 2003 Jan 8;51(1):117-31.

[10] Tadmor EB, Hai S. A Peierls criterion for the onset of deformation twinning at a crack tip. Journal of the Mechanics and Physics of Solids. 2003 May 31;51(5):765-93

[11] Gumbsch P, Beltz GE. On the continuum versus atomistic descriptions of dislocation nucleation and cleavage in nickel. Modelling and Simulation in Materials Science and Engineering.1995Sep;3(5):597.

635 [12] Zhou SJ, Carlsson AE, Thomson R. Crack blunting effects on dislocation emission from cracks. Physical review letters. 1994 Feb 7;72(6):852.

[13] Xu G, Argon AS, Ortiz M. Nucleation of dislocations from crack tips under mixed modes of loading: implications for brittle against ductile behavior of crystals. Philosophical Magazine A. 1995 Aug 1;72(2):41551.

640 [14] Juan YM, Sun Y, Kaxiras E. Ledge effects on dislocation emission from a crack tip: a first-principles study for silicon. Philosophical Magazine Letters. 1996 May 1;73(5):233-40.

[15] Andric P, Curtin WA. New Theory for Mode I Crack-tip Dislocation Emission. Journal of the Mechanics and Physics of Solids. 2017; 106:315-337.

[16] Yamakov VI, Warner DH, Zamora RJ, Saether E, Curtin WA, Glaessgen EH. Investigation of crack tip dislocation emission in aluminum using multiscale molecular dynamics simulation and continuum modeling. Journal of the Mechanics and Physics of Solids. 2014 Apr 30;65:35-53.

[17] Hirth, J.P. , Lothe, J. , 1982. The Peierls-Nabarro dislocation model. In: Theory of Dislocations. Wiley, New York, pp. 218-224. (Chapter 8.2).

[18] Mishin Y, Farkas D, Mehl MJ, Papaconstantopoulos DA. Interatomic potentials for monoatomic metals from experimental data and ab initio calculations. Physical Review B. 1999 Feb 1;59(5):3393. 
This is a post-print of the following article: Andric, Predrag; Curtin, W.A. Journal of the Mechanics and Physics of Solids 2018,, 144-161. The formal publication is available at http://dx.doi.org/10.1016/j.jmps.2018.01.016 @ 2018. This manuscript version is made available under the CC-BY-NC-ND 4.0 license http://creativecommons.org/licenses/by-nc-nd/4.0/

[19] Rice JR. A path independent integral and the approximate analysis of strain concentration by notches and cracks. 1968. ASME.

[20] Sun Y, Beltz GE. Dislocation nucleation from a crack tip: a formulation based on anisotropic elasticity. Journal of the Mechanics and Physics of Solids. 1994 Dec 1;42(12):1905-32.

655 [21] Stroh AN. Dislocations and cracks in anisotropic elasticity. Philosophical magazine. 1958 Jun 1;3(30):625-46

[22] Curtin WA. On lattice trapping of cracks. Journal of Materials Research. 1990 Jul;5(7):1549-60.

[23] Lin IH, Thomson R. Cleavage, dislocation emission, and shielding for cracks under general loading. Acta Metallurgica. 1986 Feb 1;34(2):187-206.

[24] Rice JR. Conserved integrals and energetic forces. Fundamentals of deformation and fracture. 1985:33-56.

[25] Plimpton S. Fast parallel algorithms for short-range molecular dynamics. Journal of computational physics. 1995 Mar 1;117(1):1-9.

[26] Stukowski A. Visualization and analysis of atomistic simulation data with OVITO-the Open Visualization Tool. Modelling and Simulation in Materials Science and Engineering. 2009 Dec 15;18(1):015012.

[27] Ercolessi F, Adams JB. Interatomic potentials from first-principles calculations: the force-matching method. EPL (Europhysics Letters). 1994 Jun 10;26(8):583.

[28] Mishin Y, Mehl MJ, Papaconstantopoulos DA, Voter AF, Kress JD. Structural stability and lattice defects in copper: Ab initio, tight-binding, and embedded-atom calculations. Physical Review B. 2001 May 21;63(22):224106.

[29] Adams JB, Foiles SM, Wolfer WG. Self-diffusion and impurity diffusion of fee metals using the fivefrequency model and the embedded atom method. Journal of Materials Research. 1989 Feb 1;4(01):102-12.

[30] Varvenne C, Luque A, Nöhring WG, Curtin WA. Average-atom interatomic potential for random alloys. Physical Review B. 2016 Mar 11;93(10):104201.

[31] Bonny G, Terentyev D, Pasianot RC, Poncé S, Bakaev A. Interatomic potential to study plasticity in stainless steels: the FeNiCr model alloy. Modelling and simulation in materials science and engineering. 2011 Nov 3;19(8):085008.

[32] Zamora RJ, Nair AK, Hennig RG, Warner DH. Ab initio prediction of environmental embrittlement at a crack tip in aluminum. Physical Review B. 2012 Aug 2;86(6):060101.

[33] Gumbsch P. An atomistic study of brittle fracture: toward explicit failure criteria from atomistic modeling. Journal of Materials Research. 1995 Nov;10(11):2897-907.

680 [34] Warner DH, Curtin WA. Origins and implications of temperature-dependent activation energy barriers for dislocation nucleation in face-centered cubic metals. Acta Materialia. 2009 Aug 31;57(14):4267-77.

[35] Ting TC. Anisotropic elasticity: theory and applications. Oxford University; 1996. 